\title{
Substitution Effects on a New Pyridylbenzimidazole Acceptor for Thermally Activated Delayed Fluorescence and Their Use in Organic Light-Emitting Diodes
}

\author{
David Hall, Pachaiyappan Rajamalli, Eimantas Duda, Subeesh Madayanad Suresh, \\ Francesco Rodella, Sergey Bagnich, Cameron L. Carpenter-Warren, David B. Cordes, \\ Alexandra M. Z. Slawin, Peter Strohriegl, David Beljonne, Anna Köhler,* Yoann Olivier,* \\ and Eli Zysman-Colman**
}

In this work a new acceptor is used for use in thermally activated delayed fluorescence (TADF) emitters, pyridylbenzimidazole, which when coupled with phenoxazine allows efficient TADF to occur. $\mathbf{N}$-functionalization of the benzimidazole using methyl, phenyl, and tert-butyl groups permits color tuning and suppression of aggregation-caused quenching (ACQ) with minimal impact on the TADF efficiency. The functionalized derivatives support a higher doping of 7 wt\% before a fall-off in photoluminescence quantum yields is observed, in contrast with the parent compound, which undergoes ACQ at doping concentrations greater than $1 \mathrm{wt} \%$. Complex conformational dynamics, reflected in the time-resolved decay profile, is found. The singlettriplet energy gap, $\Delta E_{\mathrm{ST}}$, is modulated by $N$-substituents of the benzimidazole and ranges of between $\mathbf{0 . 2 2}$ and $0.32 \mathrm{eV}$ in doped films. Vacuum-deposited organic light-emitting diodes, prepared using three of the four analogs, show maximum external quantum efficiencies, $\mathrm{EQE}_{\max }$, of $23.9 \%, 22.2 \%$, and $18.6 \%$ for BIm(Me)PyPXZ, BIm(Ph)PyPXZ, and BImPyPXZ, respectively, with a correlated and modest efficiency roll-off at $100 \mathrm{~cd} \mathrm{~m}^{-2}$ of $19 \% 13 \%$, and $24 \%$ of the $\mathrm{EQE}_{\text {max }}$, respectively.

\section{Introduction}

The study and design of emitters showing thermally activated delayed fluorescence (TADF) have benefitted from a surge of interest in recent years, ${ }^{[1-3]}$ with examples of organic light-emitting diodes (OLEDs) that rival their phosphorescent counterparts in terms of device efficiency. TADF involves triplet harvesting through a reverse intersystem crossing (RISC) mechanism, converting the normally nonemissive triplet states to emissive singlets. ${ }^{[4]}$ In order to maximize triplet harvesting a small singlet-triplet excited state energy gap, $\Delta E_{\mathrm{ST}}$, is required, which can be achieved by separating the hole and electron densities within the emitter by having poorly conjugated, covalently coupled donors (electron rich) and acceptors (electron deficient). ${ }^{[4]}$ When $\Delta E_{\mathrm{ST}}$ is sufficiently small, conversion of the lowest
D. Hall, P. Rajamalli, S. M. Suresh, C. L. Carpenter-Warren, D. B. Cordes, A. M. Z. Slawin, E. Zysman-Colman

Organic Semiconductor Centre

EaStCHEM School of Chemistry

University of St Andrews

St Andrews KY16 9ST, UK

E-mail: eli.zysman-colman@st-andrews.ac.uk

D. Hall, D. Beljonne

Laboratory for Chemistry of Novel Materials

University of Mons

Mons 7000, Belgium

The ORCID identification number(s) for the author(s) of this article can be found under https://doi.org/10.1002/adom.202100846.

(C) 2021 The Authors. Advanced Optical Materials published by WileyVCH GmbH. This is an open access article under the terms of the Creative Commons Attribution-NonCommercial License, which permits use, distribution and reproduction in any medium, provided the original work is properly cited and is not used for commercial purposes.
E. Duda, S. Bagnich, A. Köhler

Soft Matter Optoelectronics

BIMF and BPI

University of Bayreuth

Universitätsstraße 30, 95447 Bayreuth, Germany

E-mail: anna.koehler@uni-bayreuth.de

F. Rodella, P. Strohriegl

Macromolecular Chemistry I

$\mathrm{BIMF}$ and BPI

University of Bayreuth

95440 Bayreuth, Germany

Y. Olivier

Unité de Chimie Physique Théorique et Structurale and Laboratoire de Physique du Solide

Namur Institute of Structured Matter

Université de Namur

Rue de Bruxelles, 61, Namur 5000, Belgium

E-mail:yoann.olivier@unamur.be

DOI: 10.1002/adom.202100846 
triplet excited state $\left(\mathrm{T}_{1}\right)$ to the lowest singlet excited state $\left(\mathrm{S}_{1}\right)$ becomes possible at room temperature.

Despite the many hundreds of examples of donor-acceptor TADF emitters, most designs involve the combination of a rather limited number of classes of electron-donating and accepting groups. ${ }^{[2]}$ The most popular donors are carbazole, diphenylamine, dimethylacridine, phenoxazine (PXZ), phenothiazine, and derivatives thereof. Acceptors originate primarily from moieties containing cyano, pyridine, triazine, borane, sulfone, and ketone groups. Pyridylbenzimidazole (BImPy) has been reported previously in the literature as a fluorescent compound ${ }^{[5,6]}$ or as a ligand that coordinates to metals. ${ }^{[7-12]}$ While pyridine is an acceptor, the imidazole moiety has both electron rich and electron poor contributions ${ }^{[13]}$ allowing it to act as either an acceptor or a donor, depending on what it is coupled to. ${ }^{[13,14]}$ The use of BImPy as a component of a purely organic emitter in OLEDs has only been reported when the group is coordinated to boron, acting as a Lewis acid, producing blue fluorescent devices. ${ }^{[15]}$ TADF was detected in one example where a BImPy D-A system with a PXZ donor was coordinated to $\operatorname{Ag}(\mathrm{I}),{ }^{[16]}$ wherein the metal, again acting as a Lewis acid, contributes to a reduced $\Delta E_{\mathrm{ST}}$ in the material, thereby turning on TADF. A similar observation was made for a pyridylpyrazol D-A ligand with 9,9-dimethyl-9,10-dihydroacridine as the donor, again coordinated to $\mathrm{Ag}(\mathrm{I}) \cdot{ }^{[17]}$ In both of these examples the free ligand did not show any TADF. ${ }^{16,17]}$ To the best of our knowledge no organic TADF emitters have been reported utilizing the BImPy core alone or with a BImPy acceptor. Separately though, both pyridines and imidazoles have been investigated as acceptors in TADF emitter design. Representative examples are shown in Figure 1; several of the imidazole-containing compounds have been reported to emit by either triplet-triplet annihilation ${ }^{[18]}$ or hot exciton mechanisms ${ }^{[13,19]}$ in lieu of TADF. A summary of the structures of purely organic pyridinecontaining TADF emitters and their properties can be found in Figure S1 and Table S1 in the Supporting Information while those of imidazole-containing emitters are reported in Figure S2 and Table S2 in the Supporting Information.

Herein we report a series of structurally related functionalized BImPy TADF emitters containing a phenoxazine donor (PXZ). The parent emitter, BImPyPXZ, contains no $N$-substitution while methyl (BIm(Me)PyPXZ), phenyl (BIm(Ph) PyPXZ), and tert-butyl (BIm(tBu)PyPXZ) derivatives present ever increasing torsions due to the increasing steric bulk of the $N$-substituent. The increased bulkiness of these groups was introduced as a strategy to aid in the suppression of aggregation-caused quenching (ACQ), ${ }^{[20]}$ a common nonradiative pathway observed in films of emitters. ${ }^{[21]}$ Each of the emitters displays TADF behavior in the solid state, with $\Delta E_{\mathrm{ST}}$ of between 0.22 and $0.32 \mathrm{eV}$ in doped mCP films. Green-emitting OLEDs were fabricated using BImPyPXZ, BIm(Me)PyPXZ, and BIm(Ph)PyPXZ, and show excellent maximum external quantum efficiencies, $\mathrm{EQE}_{\max }$, of $18.6 \%, 23.9 \%$, and $22.2 \%$, respectively.

\section{Results and Discussion}

\subsection{Synthesis}

Each of the emitters was obtained following a multistep synthesis as documented in Figure 2. 5-Bromopyridine-2-carbaldehyde was first protected as its acetal, 1, in an excellent yield of 95\%. Protection is essential for the subsequent crosscoupling step; direct crosscoupling was not possible, which we attributed to metal binding between the oxygen and nitrogen atoms of the substrate. A Buchwald-Hartwig crosscoupling reaction installed the phenoxazine donor onto the pyridine (2) in a good yield of $83 \%$. Removal of the acetal under acidic conditions afforded a red-colored compound, PXZPyCHO, which itself was weakly emissive in solution and in the solid state (vide infra). Functionalized 2-nitroanilines were synthesized via $\mathrm{S}_{\mathrm{NAr}}$ reactions
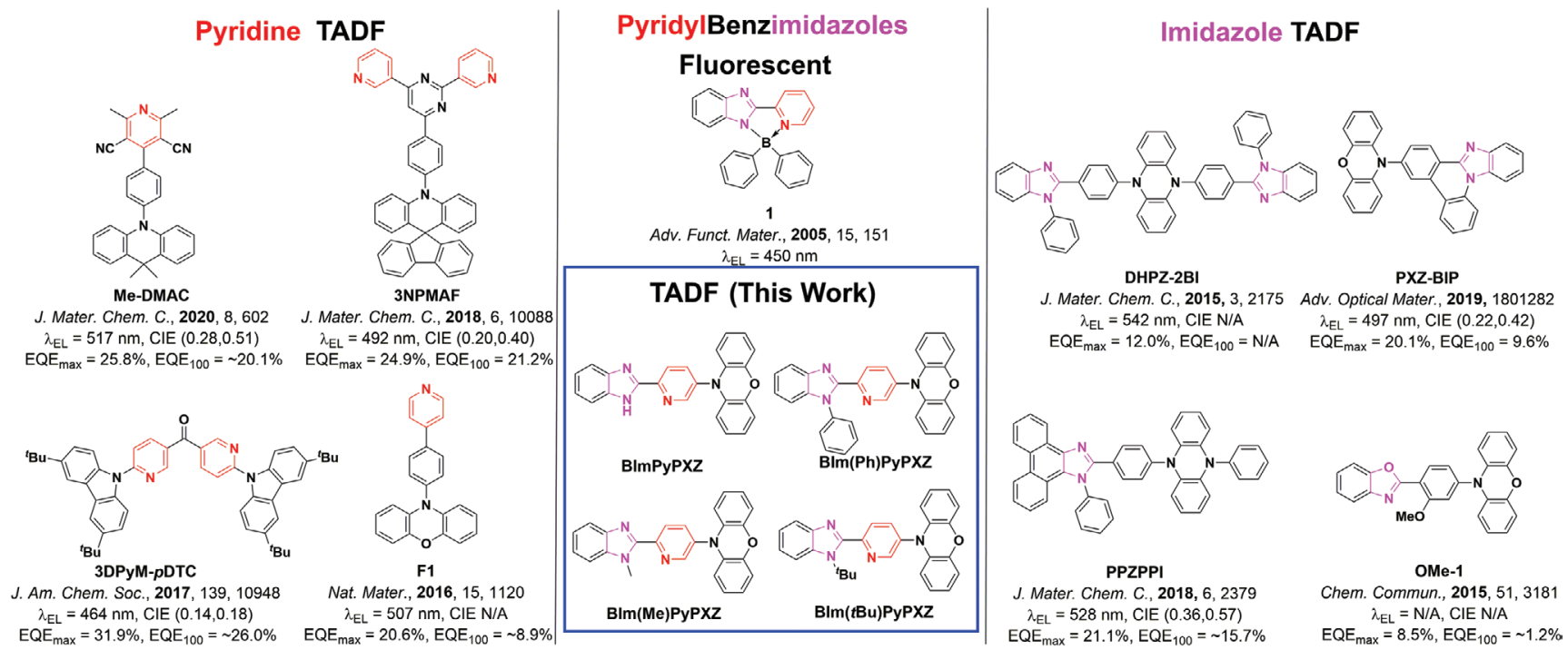

Figure 1. Structures of some of the previously reported pyridine- and imidazole-containing TADF emitters with the highest efficiencies along with the structure of the only purely organic example of pyridylbenzimidazoles being used in OLEDs as well as the structures of the emitters reported in this work. The use of the $\sim$ indicates that the data were extracted from the figure in the literature report. 

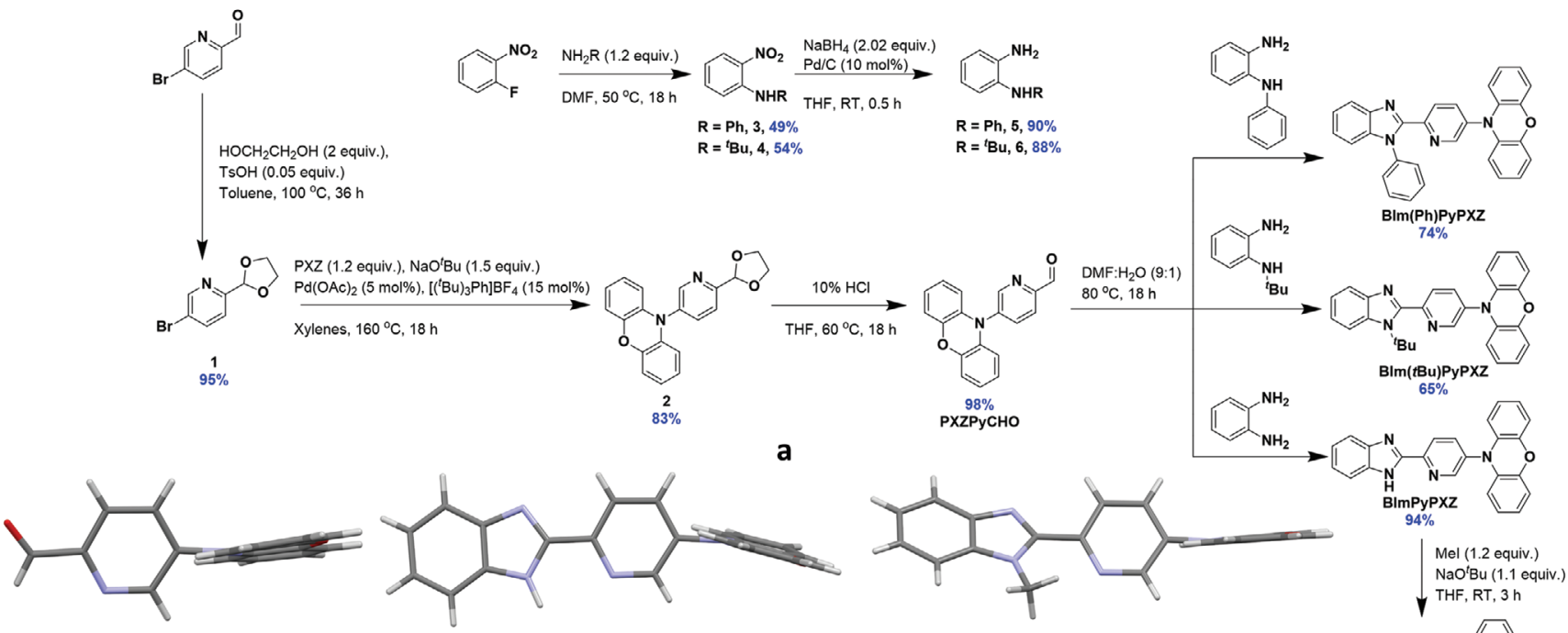

b

C
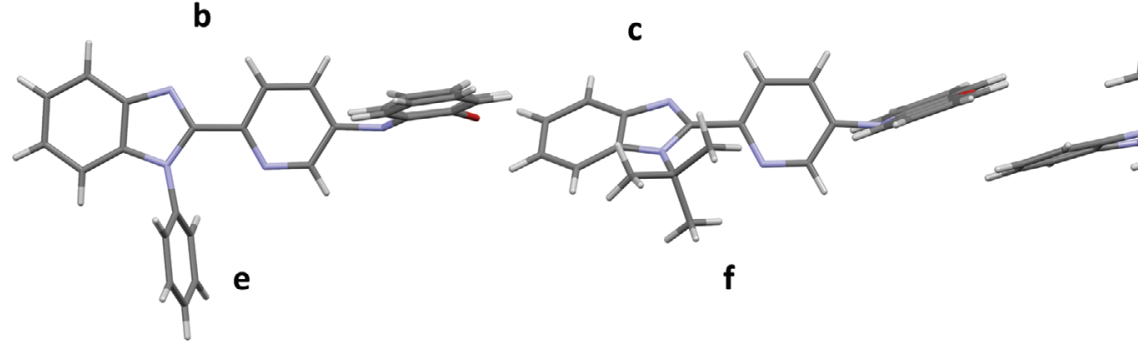

d
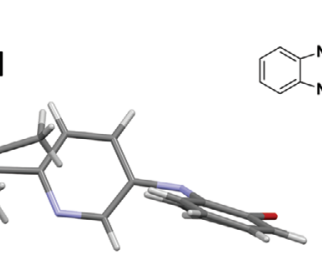

$\checkmark$ THF, RT, $3 \mathrm{~h}$ (t)

Figure 2. a). Synthesis of the four targets and crystal structures of b) PXZPyCHO, c) BImPyPXZ, d) BIm(Me)PyPXZ, e) BIm(Ph)PyPXZ, f) BIm(tBu) PyPXZ with planar PXZ, and g) BIm(tBu)PyPXZ with a puckered PXZ.

of 2-fluoronitrobenzene using tert-butylamine or aniline in reasonable yields (see the Supporting Information for details). Subsequent reduction afforded the relevant functionalized diaminobenzenes in good yield. Following the literature, condensation of these intermediates with PXZPyCHO produced BImPyPXZ, BIm(Ph)PyPXZ, and BIm(tBu)PyPXZ in yields of between $65 \%$ and $94 \% .{ }^{[22]}$ The last target compound, BIm(Me) PyPXZ, was synthesized via methylation of BImPyPXZ using MeI in $96 \%$ yield.

Purification by temperature-gradient vacuum sublimation afforded pure BImPyPXZ, BIm(Me)PyPXZ, and BIm(Ph) PyPXZ; however, cleavage of the $N$-tBu bond in $\operatorname{BIm}(t \mathrm{Bu})$ PyPXZ occurred under these conditions, thus preventing its use in vacuum-deposited OLEDs. Crystals suitable for single crystal X-ray diffraction were grown for BImPyPXZ, BIm(Me) PyPXZ, BIm(Ph)PyPXZ, BIm(tBu)PyPXZ, and PXZPyCHO. Both BIm(Ph)PyPXZ and BImPyPXZ were grown via slow vapor diffusion of hexane into a saturated solution of toluene. BIm(Me)PyPXZ was grown from a saturated solution of chloroform layered with ethanol. BIm(tBu)PyPXZ was grown from a saturated solution of dichloromethane layered with hexane. PXZPyCHO was grown via slow evaporation of a saturated solution of toluene. All the structures displayed similar PXZ-Py torsions, ranging from $60.0(15)^{\circ}$ to $89.72(17)^{\circ}$. The large torsion is often observed in TADF emitters with PXZ donors owing to its large size, with some variation arising from the degree of pucker and how this interacts with the pyramidalization of the PXZ nitrogen. ${ }^{[23]}$ PXZPyCHO and BIm(Me)PyPXZ both contain a planar PXZ, with $\pi$-stacking interactions occurring between cofacial PXZ groups (Figures S40 and S44, Supporting Information), resulting in $\pi$-stacked chains along the $a$-axes. The structure of BImPyPXZ shows PXZ donors with a range of slightly puckered conformations (angles between PXZ phenyl ring planes $5.4^{\circ}-12.3^{\circ}$, Figure S45, Supporting Information). Given the closeness to planarity in this arrangement, it is somewhat surprising to see only one $\pi$-stacking interaction occurring between PXZ donor groups. The primary intermolecular interactions in this compound are hydrogen bonds between imidazole moieties, giving rise to chains along the $b$-axis. In contrast, the PXZ donor in $\mathbf{B I m}(\mathbf{P h}) \mathbf{P y P X Z}$ has a decidedly puckered conformation (angle between PXZ phenyl ring planes $23.0^{\circ}$, Figure S43, Supporting Information), similar to that previously observed for phenothiazine donors. ${ }^{[24]}$ In $\operatorname{BIm}(t \mathrm{Bu}) \mathbf{P y P X Z}$, two different PXZ conformers were observed in the same structure, one with a planar PXZ (Figure 2f) and the other adopting an intermediate puckered shape (angle between PXZ phenyl ring planes $16.5^{\circ}$, Figure $2 \mathrm{~g}$ ). No $\pi-\pi$ interactions are observed between planar PXZ moieties in this compound. There is no consistent trend to suggest that particular solid-state intermolecular interactions drive a tendency for planar versus puckered PXZ groups. The continuum seen between strictly planar, through various intermediate to more severely puckered forms of PXZ suggest a subtle source for this, possibly involving intermolecular $\pi \cdots \pi$ and $\mathrm{CH} \cdots \pi$ interactions, and likely low energetic differences between different conformations. The next torsion of importance is $\alpha$, the torsion between the benzimidazole 
and the substituted pyridine (Figure S41, Supporting Information). This torsion will govern the conjugation length of the acceptor. The different compounds show three ranges of torsions, broadly corresponding to the steric bulk of the $N$-substituent within the plane of the benzimidazole. These result in near-planar arrangements of the two rings for BImPyPXZ (Figure S42, Supporting Information) and BIm(Ph)PyPXZ (Figure S43, Supporting Information) [torsions of $1.6(8)^{\circ}$ $12.4(8)^{\circ}$ and $4.59(19)^{\circ}$, respectively], an intermediate arrangement for BIm(Me)PyPXZ (Figure S44, Supporting Information) [torsion of $23.9(2)^{\circ}$, and the rings tending toward orthogonality for BIm( $t \mathrm{Bu})$ PyPXZ (Figure S45, Supporting Information).

Thermogravimetric analysis (TGA) and differential scanning calorimetry (DSC) were performed to assess the thermal properties of the four emitters (Figures S46-S49 and Table S4, Supporting Information). BImPyPXZ, BIm(Me)PyPXZ, and BIm(Ph)PyPXZ showed similar TGA behavior with temperatures $T_{\mathrm{d}}$, of $5 \%$ weight loss of 346,341 , and $355^{\circ} \mathrm{C}$, respectively, indicative of their high thermal stability. The fact that the weight drops to $0 \%$ shows that the three compounds do not in fact decompose but completely sublime already at atmospheric pressure during the TGA experiment. The $T_{\mathrm{d}}$ of $276{ }^{\circ} \mathrm{C}$ is significantly lower for $\mathbf{B I m}(t \mathbf{B u})$ PyPXZ indicating that the benzimidazole with the tert-butyl substituent is not thermally stable, which was also apparent during attempted vacuum sublimation.

BImPyPXZ shows a glass transition temperature $\left(T_{\mathrm{g}}\right)$ of $97{ }^{\circ} \mathrm{C}$ in the DSC experiment. The material recrystallizes at about $160{ }^{\circ} \mathrm{C}$ and finally melts at $260{ }^{\circ} \mathrm{C}$. BIm(Me)PyPXZ exhibits a glass transition at $64{ }^{\circ} \mathrm{C}$. Its melting behavior is rather complex and the occurrence of two melting temperatures in the first and second heating cycle in Figure S47b in the Supporting Information points to the formation of two polymorphs. This is further supported when the material is investigated using different cooling rates with slow and fast temperature ramps as shown in Figure S49 in the Supporting Information. Here, the melting points of both polymorph 1 at $225^{\circ} \mathrm{C}$ and of polymorph 2 at $210{ }^{\circ} \mathrm{C}$ become visible. The $T_{\mathrm{g}}$ of $\mathrm{BIm}(\mathrm{Ph}) \mathrm{PyPXZ}$ is $73{ }^{\circ} \mathrm{C}$. In the first heating cycle, the material is observed to melt at $162{ }^{\circ} \mathrm{C}$. Upon cooling, no recrystallization occurs and, in the $2 \mathrm{nd}$, 3rd, and 4th heating only a $T_{\mathrm{g}}$ is observed. Due to the thermal instability of $\operatorname{BIm}(t \mathbf{B u}) \operatorname{PyPXZ}$ the DSC experiments have to be interpreted with great care. From Figure S48d in the Supporting Information only a $T_{g}$ of about $80^{\circ} \mathrm{C}$ can be estimated.

\subsection{Theoretical Modeling}

Ground state geometry optimization using density functional theory (DFT) was performed on each of the emitters using the M062X functional in combination with the 6-31G(d,p) basis set (Figure 3). Vertical excitation calculations from the ground state geometry at the time-dependent DFT (TD-DFT) within the Tamm-Dancoff approximation (TDA-DFT) using the aforementioned functional and basis set were also carried out. ${ }^{[25,26]}$ TDA-DFT calculations are preferentially employed with respect to TD-DFT because they address the triplet instability issue, which tends to overstabilize the triplet states bearing a strong locally excited (LE) state character. ${ }^{[25]}$ M062X was selected over the widely used PBE0 and B3LYP functionals since the latter two tend to overstabilize excited states containing a significant amount of charge transfer (CT) character due to their small content of Hatree-Fock exchange. ${ }^{[26]}$

In the ground state, changing the $\mathrm{R}$ group alters both the equilibrium torsion angle $\alpha$ and bond length $\mathrm{C}_{\gamma}-\mathrm{C}_{\gamma}$ (Figure S50 and Table S5, Supporting Information). Both BImPyPXZ and BIm(Me)PyPXZ have a predicted ground state geometry that is planar $\left(\alpha=0^{\circ}\right)$ likely stabilized due to a combination of increased conjugation and intramolecular hydrogen bonding. ${ }^{[27]}$ The weakly inductive electron-donating ability of the methyl substituent increases the Lowest Unoccupied Molecular Orbital (LUMO) energy compared to the parent BImPyPXZ. A similar observation was reported previously for fluorescent imidazolebased emitters. ${ }^{[28]}$ Owing to the larger size of the $\mathrm{Ph}$ and $t \mathrm{Bu}$ substituents compared to the parent and methylated structures, the torsion angle, $\alpha$, deviates from the usually favorable $\alpha=0^{\circ}-19.5^{\circ}$ and $40.8^{\circ}$, respectively, which in turn disrupts conjugation between the BIm and Py heterocycles, increasing the $\mathrm{C}_{\mathrm{BIm}}-\mathrm{C}_{\mathrm{Py}}$ bond length slightly (Table S5, Supporting Information). A consequence of this conformational change is a predicted increase in the LUMO energy. The destabilization is more pronounced in $\mathbf{B I m}(\mathbf{t B u}) \mathbf{P y P X Z}$ as the inductively electron-withdrawing phenyl group in $\mathbf{B I m}(\mathbf{P h}) \mathbf{P y P X Z}$ counteracts the decrease in conjugation due to this bulky substituent. ${ }^{[28]}$ In each example there is very little change in the Highest Occupied Molecular Orbital (HOMO) level, which is localized on the PXZ. HOMO to LUMO transitions dominate vertical excitations to the $\mathrm{S}_{1}$ while the $\mathrm{T}_{1}$ state is a more complex picture involving several different transitions (Table S7, Supporting Information). The overall increase in the HOMO-LUMO energy gap for BIm(Me)PyPXZ compared to BImPyPXZ translates also in an increased $\mathrm{S}_{1}$ energy level from 3.53 to $3.58 \mathrm{eV}$. Similarly, the $\mathrm{S}_{1}$ energy increases from $\mathbf{B I m}(\mathbf{P h}) \mathbf{P y P X Z}(3.59 \mathrm{eV})$ to $\mathbf{B I m}(\boldsymbol{t B u})$ PyPXZ $(3.69 \mathrm{eV})$. Calculated $\Delta E_{\mathrm{ST}}$ values remain small at 0.05, 0.11, 0.09, and $0.15 \mathrm{eV}$ for BImPyPXZ, BIm(Me)PyPXZ, $\operatorname{BIm}(\mathbf{P h}) \mathbf{P y P X Z}$, and $\mathrm{BIm}(t \mathrm{Bu}) \mathrm{PyPXZ}$, respectively. The TADF efficiency is governed not only by the small $\Delta E_{\mathrm{ST}}$ but also by the magnitude of the spin-orbit coupling (SOC) between the $\mathrm{S}_{1}$ and $\mathrm{T}_{1}$ states. Adherence to El Sayed's rules requires that the nature of $\mathrm{T}_{1}$ and $\mathrm{S}_{1}$ be different for state mixing to occur; ${ }^{[29]}$ transitions between two purely CT or LE states are strictly forbidden. ${ }^{[30]}$ Several groups ${ }^{[30-32]}$ have highlighted that the presence of intermediate triplet states of a differing nature to those of $\mathrm{S}_{1}$ can be recruited to bypass the unfavorable $\mathrm{T}_{1}-\mathrm{S}_{1}$ SOC-driven conversion. In this case, RISC occurs through a spin-vibronic mechanism, namely the $\mathrm{T}_{1}-\mathrm{S}_{1}$ conversion is a two-step mechanism involving reverse internal conversion between $T_{1}$ and a higher-lying triplet state $\mathrm{T}_{n}$ followed by an SOC-driven spin RISC between $\mathrm{T}_{n}$ and $\mathrm{S}_{1} \cdot{ }^{[32,33]}$ We thus quantified the degree of charge-transfer character of the excited state by computing the $\phi_{\mathrm{S}}$ metric through postprocessing of the TDA-DFT calculations performed here. ${ }^{[34]}$ The $\phi_{\mathrm{S}}$ metric is a measure of the overlap the electronic density of the frontier orbitals during the electronic transition. A $\phi_{\mathrm{S}}$ value of 1 represents an LE state while a value of 0 corresponds to a purely CT excited state, intermediate values are interpreted as mixed CT-LE excited states. The $\mathrm{S}_{1}$ state of each emitter is predominantly CT in nature 


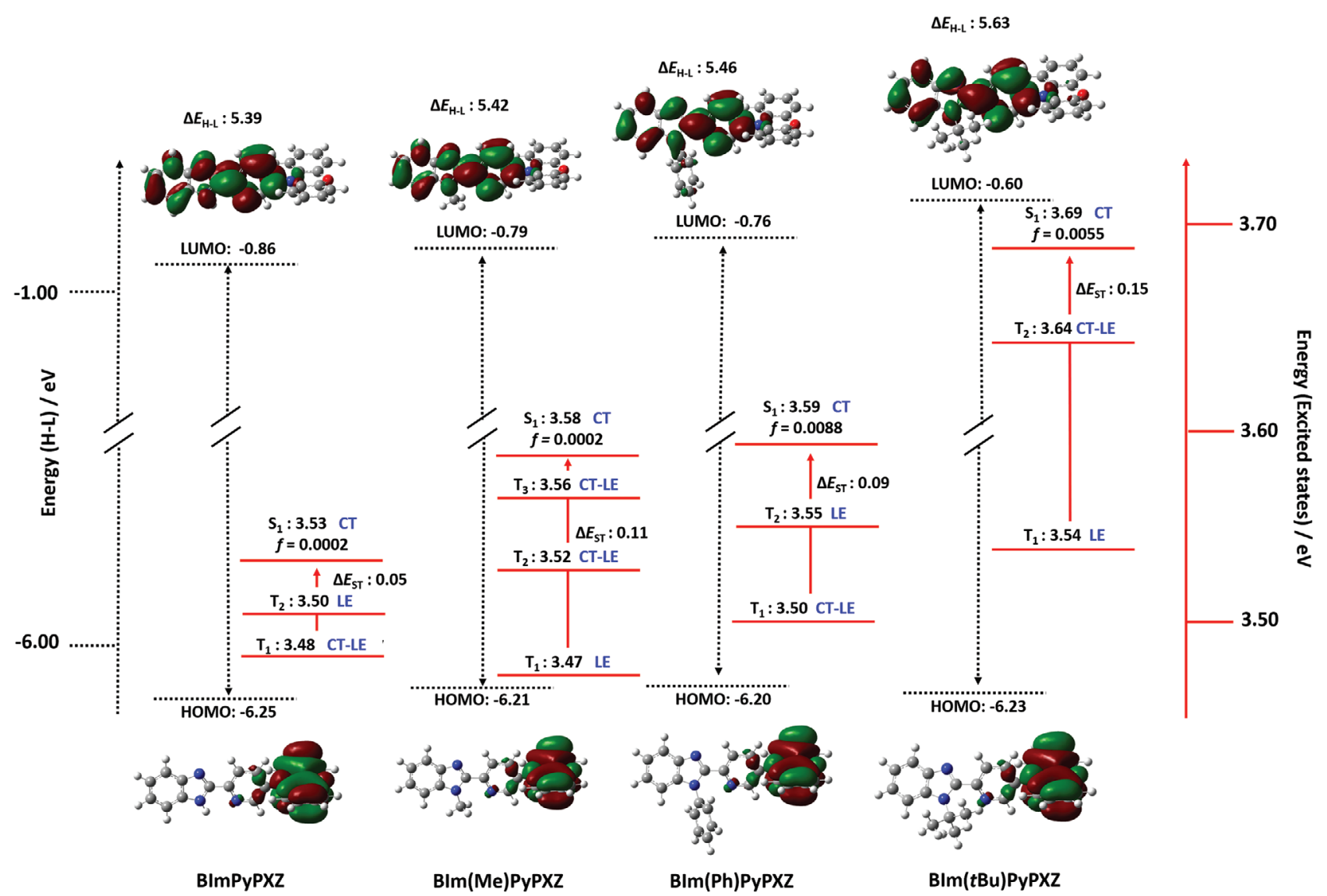

Figure 3. TDA-DFT calculated HOMO LUMO levels (dotted lines) and excited triplet and singlet state energies (solid lines), and electron density distribution plots of the HOMO and LUMO calculated using M062X//6-31G (d,p). The nature of singlet and triplet excited states is indicated in blue based on the $\phi_{\mathrm{S}}$ values of overlap where $\mathrm{CT}=0, \mathrm{LE}=1$ and $\mathrm{CT}-\mathrm{LE}=0.33-0.66$ (isovalue $=0.02$ ).

$\left(\phi_{\mathrm{S}}<0.33\right)$. This is a common occurrence for donor-acceptor TADF emitters and this assignment is supported by their positive solvatochromism (vide infra). The nature of $\mathrm{T}_{1}$ varies, being mixed CT-LE for BImPyPXZ (0.48) and BIm(Ph)PyPXZ (0.65), and mostly LE for BIm(Me)PyPXZ (0.82) and BIm(tBu)PyPXZ (0.76). The intermediate states are of mixed CT-LE character for BIm(Me)PyPXZ (0.67 and 0.57) and BIm(tBu)PyPXZ $(0.60)$ and LE for BImPyPXZ (0.82) and BIm(Ph)PyPXZ (0.81), which is the opposite trend to that observed for the nature of the $T_{1}$ state. These intermediate states of differing character to each other and to the $S_{1}$ state are expected to facilitate RISC due to spinvibronic coupling. ${ }^{[30,35]}$ A summary of the excited state energies and properties is found in Table S6 and Figure S51 in the Supporting Information. The intermediate, PXZPyCHO, was also investigated computationally and presents a very small $\Delta E_{\mathrm{ST}}$ of $0.06 \mathrm{eV}$, suggestive that it too will show TADF. Its $\mathrm{S}_{1}$ energy is lower than that of the BImPyPXZ series owing to the stronger electron-acceptor in this compound (Figure S54 and Tables S5 and S6, Supporting Information).

In the crystal structures, significantly different dihedral angles $\alpha$ between the pyridyl and benzimidazole units are observed for several of the compounds, caused by differences in local inter- and intramolecular interactions. In particular, for $\operatorname{BIm}(t \mathrm{Bu}) \mathbf{P y P X Z}$, we found two different conformers with torsion angles of $47.16(19)^{\circ}$ and $77.98(18)^{\circ}$. In order to probe the significance of torsion around $\alpha$ we computed the torsion potential about the $\mathrm{C}_{\mathrm{BIm}}-\mathrm{C}_{\mathrm{Py}}$ bond (Figure 4 ) for each emitter, varying the $\alpha$ dihedral angle in increments of $5^{\circ}$. Each conformer was reoptimized at M062X//6-31G(d,p) level in the ground state. Vertical excited state TDA-DFT calculations were then carried out for each ground-state conformer where we investigated the excited-state properties including $\Delta E_{\mathrm{ST}}$, emission energy and the nature of the excited states. There are relatively few reports that systematically correlate the effects of conformation on the optoelectronic properties of TADF emitters. ${ }^{[33,34,36]}$ Here we applied a Boltzmann population distribution analysis to assess the contribution at room temperature of the different conformers to the photophysical landscape (Figure 4). For BImPyPXZ, we observe a large increase in energy as conjugation is disrupted from the optimized conformation with $\alpha=0^{\circ}$, with a barrier to rotation occurring at $90^{\circ}$ at $\approx 12 \mathrm{kcal} \mathrm{mol}^{-1}$. There is a second, smaller rotational barrier of $\approx 10 \mathrm{kcal} \mathrm{mol}^{-1}$ at $180^{\circ}$ due to steric interaction between $\mathrm{NH}$ and $\mathrm{CH}$ hydrogen atoms on the adjacent heterocycles (Figure 4a). The rotational profile for BIm(Me)PyPXZ is similar with an energy minimum at $\alpha=0^{\circ}$, although the largest barrier of $\approx 11 \mathrm{kcal} \mathrm{mol}^{-1}$ occurs at $180^{\circ}$ due to steric hindrance between the proton and the methyl group on the Py and BIm units, respectively (Figure 4b). The lowest energy conformer for 

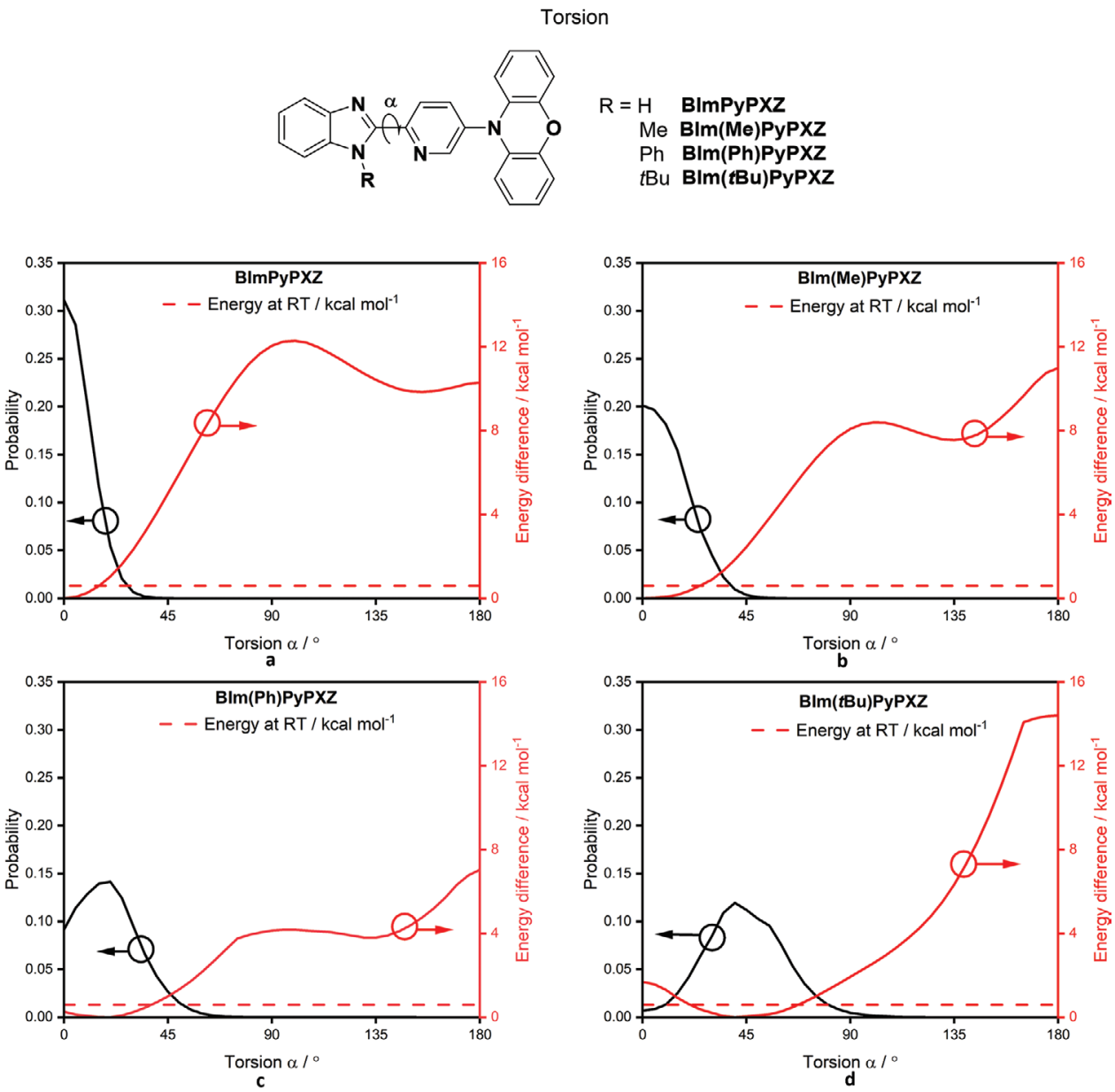

Figure 4. Boltzmann probability distribution and energy difference from lowest energy conformer calculated around torsion $\alpha$ for a) BImPyPXZ, b) $\operatorname{BIm}(\mathrm{Me}) \mathrm{PyPXZ}, \mathrm{c}) \mathrm{BIm}(\mathrm{Ph}) \mathrm{PyPXZ}$, and d) BIm(tBu)PyPXZ. Calculated at M062X//6-31G (d,p) level of theory in the gas phase with dispersion effects included. The dashed horizontal line represents the energy at room temperature.

$\operatorname{BIm}(\mathrm{Ph}) \mathrm{PyPXZ}$ occurs at $\alpha=20^{\circ}$ and its rotational barrier is the lowest amongst the four compounds, peaking at $\approx 7 \mathrm{kcal} \mathrm{mol}^{-1}$ at $180^{\circ}$ (Figure 4c). Finally, the steric interactions of the tert-butyl group in $\operatorname{BIm}(t \mathrm{Bu})$ PyPXZ have more effect on both $\alpha$, which now is $40^{\circ}$ for the lowest energy conformer, and the barrier to rotation, which is $\approx 14 \mathrm{kcal} \mathrm{mol}^{-1}$, centered at $180^{\circ}$ (Figure $4 \mathrm{~d}$ ). In most of the examples in the present study the computed value of $\alpha$ in the lowest energy conformation and the value of $\alpha$ derived from the crystal structure deviate significantly $\left(\Delta \alpha>15^{\circ}\right)$, likely due to the significant influence of both intra- and intermolecular interactions in the crystal exert on the conformational landscape. A summary of these data can be found in Table S9 in the Supporting Information, crystal packing forces are the primary causes for the discrepancy.

We computed vertical excitations energies for $S_{1}$ and $T_{1-3}$ excited states and their respective natures as well as the $\Delta E_{\mathrm{ST}}$, varying the torsion angle, $\alpha$, within the ground state torsion energy potential calculated previously. For each compound, the hole density in the $S_{1}$ excited state is localized primarily on the PXZ unit with some overlap on the Py ring. A similar trend is observed for $T_{1}$ wherein it is located primarily on the PXZ except in cases where the conformation is coplanar $\left(0^{\circ}\right.$ and $180^{\circ}$ for $\operatorname{Imid}(\mathrm{Me}) \mathrm{PyPXZ}$, and $\operatorname{Imid}(\boldsymbol{t B u}) \mathrm{PyPXZ}$, and $180^{\circ}$ for Imid(Ph)PyPXZ) where it is located across the whole BImPy unit. For each emitter the electron density is located across the BImPy component at $0^{\circ}$ and $180^{\circ}$; however, with increasing torsion the conjugation is broken, with electron density now localized mostly on the Py ring, with only modest contribution from the PXZ (Figures S57-S60, Supporting Information). Interestingly, $\Delta E_{\mathrm{ST}}$ is a maximum at $90^{\circ}$, which correlates with a maximum in $\mathrm{S}_{1}$ and $\mathrm{T}_{1}$ energies. This corresponds to a maximized hole and electron overlap near the PXZ unit and high $\phi_{\mathrm{S}}$ value for both $\mathrm{T}_{1}$ and $\mathrm{S}_{1}$. Overall, $\mathrm{T}_{1}$ energy fluctuates much less than $S_{1}$. This is essentially due to higher LE character of $\mathrm{T}_{1}$ in contrast to the high CT character of $\mathrm{S}_{1}$. Table 1 summarizes the conformational analysis of the Boltzmann-averaged excited state energies based on their population distribution, $\phi_{\mathrm{S}}, \Delta E_{\mathrm{ST}}$, oscillator strength for each emitter. When comparing Table 1 and Table S6 in the Supporting Information it is clear that changing $\alpha$ actually has a very minimal impact on these parameters. The changes in the energies of $\mathrm{S}_{1}$ and $\mathrm{T}_{2}$ as a function of dihedral angle roughly mirror each other and so $\Delta E_{\mathrm{ST} 2}$ remains rather insensitive to changes in $\alpha$. The standard deviations of each of the excited state energies, $\phi_{\mathrm{S}}$ values and $\Delta E_{\mathrm{ST}}$ 
Table 1. Boltzmann averaged vertical excitation energies and $\phi_{\mathrm{S}}$ values calculated in the gas phase within the TDA using M062X//6-31G (d,p) including dispersion correction over a range of conformations with $\alpha$ running from $0^{\circ}$ to $180^{\circ}$ at $5^{\circ}$ intervals. The values in parentheses are the standard deviations on the different parameters computed.

\begin{tabular}{lccccccccc}
\hline Compound & $\mathrm{T}_{1}[\mathrm{eV}]$ & $\mathrm{T}_{2}[\mathrm{eV}]$ & $\mathrm{T}_{3}[\mathrm{eV}]$ & $\mathrm{S}_{1}[\mathrm{eV}]$ & $\phi_{\mathrm{S}} \mathrm{T}_{1}$ & $\phi_{\mathrm{S}} \mathrm{T}_{2}$ & $\phi_{\mathrm{S}} \mathrm{T}_{3}$ & $\phi_{\mathrm{S}} \mathrm{S}_{1}$ & $\Delta E_{\mathrm{ST}}[\mathrm{eV}]$ \\
\hline BImPyPXZ & $3.46(0.02)$ & $3.52(0.02)$ & $3.57(0.02)$ & $3.54(0.01)$ & $0.58(0.07)$ & $0.80(0.02)$ & $0.72(0.02)$ & $0.26(0.01)$ & $0.08(0.02)$ \\
BIm(Me)PyPXZ & $3.48(0.03)$ & $3.54(0.03)$ & $3.59(0.04)$ & $3.60(0.02)$ & $0.75(0.05)$ & $0.75(0.06)$ & $0.64(0.06)$ & $0.27(0.01)$ & $0.11(0.01)$ \\
$\operatorname{BIm}(\mathrm{Ph}) \operatorname{PyPXZ}$ & $3.48(0.05)$ & $3.54(0.03)$ & $3.63(0.09)$ & $3.59(0.03)$ & $0.65(0.04)$ & $0.76(0.07)$ & $0.76(0.05)$ & $0.29(0.03)$ & $0.11(0.04)$ \\
BIm(tBu)PyPXZ & $3.53(0.03)$ & $3.65(0.08)$ & $3.81(0.15)$ & $3.71(0.07)$ & $0.75(0.03)$ & $0.59(0.14)$ & $0.77(0.10)$ & $0.29(0.01)$ & $0.19(0.06)$ \\
\hline
\end{tabular}

were also calculated across the range of torsion angles, which are indicated by the values in brackets in Table 1 . We note that the standard deviations of the $\mathrm{T}_{1}, \mathrm{~T}_{2}$, and $\mathrm{S}_{1}$ excited state energies as well as their corresponding $\phi_{\mathrm{S}}$ are small for all compounds with the exception of $\operatorname{BIm}(t \mathbf{B u}) \operatorname{PyPXZ}$. We stress that the excited state energies and their nature are rather insensitive with respect to the $\alpha$ torsion angle.

\subsection{Optoelectronic Properties}

The electrochemical behavior in acetonitrile of each emitter was investigated using cyclic voltammetry (CV) and differential pulse voltammetry (DPV) (Figure 5a and Table S11, Supporting Information). Each of the emitters displayed nearly identical reversible oxidation waves with values between 0.74 and $0.76 \mathrm{~V}$ vs SCE (Saturated Calomel Electrode), originating from oxidation of the PXZ donor. There is negligible impact on the oxidation potential from the presence or absence of $\mathrm{N}$-substitution on the benzimidazole acceptor. Reduction waves involving the BImPy acceptor are irreversible, with the reduction potentials varying as a function of both the inductive effect of the $\mathrm{N}$-substituent and the degree of conjugation present within the BImPy acceptor, ranging from -1.82 to $-2.22 \mathrm{~V}$ when conjugation is greatest in BImPyPXZ and smallest for $\operatorname{BIm}(t \mathrm{Bu})$ PyPXZ, respectively. The corresponding HOMO/LUMO levels are shown in Table 2 . The changing $\Delta E_{\mathrm{H}-\mathrm{L}}$ values are in good agreement with DFT (Table S5, Supporting Information, and Figure 3), with an increase in the gap resulting mainly from destabilization of the LUMOs while the HOMO values remain largely unchanged as these are situated predominately on the PXZ (calculated to be -6.25 and $-6.20 \mathrm{eV}$ and measured between -5.18 and $-5.16 \mathrm{eV}$ ). The electrochemical data of PXZPyCHO are shown in Figure S62 and Table S11 in the Supporting Information. The strongly electron-withdrawing nature of the aldehyde stabilizes the LUMO to $-2.92 \mathrm{eV}$, reducing the HOMO-LUMO gap significantly to $2.28 \mathrm{eV}$ in agreement with the calculated trend (Table S5, Supporting Information).

The UV-vis absorption spectra in toluene are shown in Figure 5b, which qualitatively agree with the simulated absorption spectra (Figure S52, Supporting Information). Each emitter shows a low energy band around $400 \mathrm{~nm}$, with molar absorptivity values, $\varepsilon$, of $2000-3000 \mathrm{M}^{-1} \mathrm{~cm}^{-1}$ associated with a transition to a mixed ${ }^{1} \mathrm{CT}$-LE state with a predominant CT character; the hole density is situated on PXZ while the electron density is located on both PXZ and Py rings (Figure S53, Supporting Information). A second high-intensity ( $\varepsilon$ of $19000-35000 \mathrm{M}^{-1} \mathrm{~cm}^{-1}$ ) band at between 300 and $333 \mathrm{~nm}$ is assigned to an LE transition with the hole and electron densities completely localized on the BImPy unit (Figure S53, Supporting Information). Both of these absorption bands in $\operatorname{BIm}(t \mathrm{Bu}) \mathbf{P y P X Z}$ are blueshifted with respect to the other compounds because of its more twisted conformation resulting in a decrease of the conjugation length within the acceptor group. The decrease in the molar absorptivity is likewise explained by the increasing bulkiness of the peripherical substituents when comparing $\mathrm{BImPyPXZ}$ to $\mathrm{BIm}(\boldsymbol{t} \mathrm{Bu}) \mathrm{PyPXZ}$, which is related to the more twisted conformation about the $\mathrm{C}_{\mathrm{BIm}}-\mathrm{C}_{\mathrm{Py}}$ bond
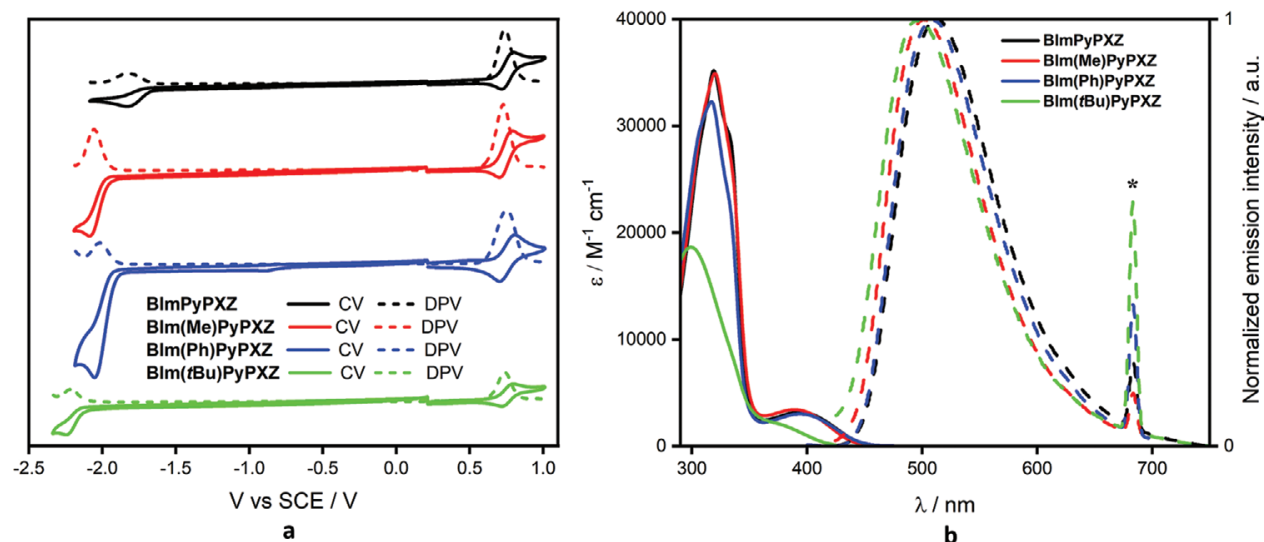

Figure 5. Solution optoelectronic spectra of the four emitters. a) CV and DPV in $\mathrm{N}_{2}$-saturated $\mathrm{MeCN}_{\text {with }} 0.1 \mathrm{M}\left[n \mathrm{nBu}_{4} \mathrm{~N}\right] \mathrm{PF} \mathrm{F}_{6}$ as the supporting electrolyte and $\mathrm{Fc} / \mathrm{Fc}^{+}$as the internal reference $(0.38 \mathrm{~V} \text { vs SCE})^{[1]}$ and b) absorption (solid) and normalized emission (dashed) spectra in dilute toluene solution, where * indicates the transmission of the excitation pulse, $\lambda_{\mathrm{exc}}=340 \mathrm{~nm}$, by the second order diffraction of the spectrometer's grating. 
Table 2. Summary of the solution-state optoelectronic properties.

\begin{tabular}{|c|c|c|c|c|c|c|c|c|}
\hline Compound & $\lambda_{\mathrm{PL}}{ }^{\mathrm{a})}[\mathrm{nm}]$ & $\Phi_{\mathrm{PL}}{ }^{\mathrm{b})}[\%]$ & $\mathrm{S}_{1}^{\mathrm{c})}[\mathrm{eV}]$ & $\mathrm{T}_{1}^{\mathrm{d})}[\mathrm{eV}]$ & $\Delta E_{\mathrm{ST}}{ }^{\mathrm{e})}[\mathrm{eV}]$ & HOMO $^{\text {ff }}[\mathrm{eV}]$ & LUMO $^{f}[\mathrm{eV}]$ & $\Delta E_{\left.\mathrm{H}-\mathrm{L}^{\mathrm{g}}\right)}[\mathrm{eV}]$ \\
\hline BImPyPXZ & 513 & 31 & 2.74 & 2.62 & 0.12 & -5.16 & -2.60 & 2.56 \\
\hline BIm(Me)PyPXZ & 501 & 36 & 2.93 & 2.64 & 0.29 & -5.16 & -2.37 & 2.79 \\
\hline BIm(Ph)PyPXZ & 509 & 40 & 2.86 & 2.63 & 0.23 & -5.18 & -2.40 & 2.78 \\
\hline $\operatorname{BIm}(t \mathrm{Bu}) \mathrm{PyPXXZ}$ & 497 & 25 & 3.11 & 2.78 & 0.33 & -5.16 & -2.20 & 2.96 \\
\hline
\end{tabular}

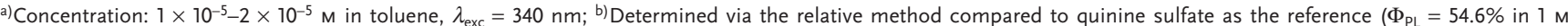
$\mathrm{H}_{2} \mathrm{SO}_{4}$ ) under $\mathrm{N}_{2}, \lambda_{\text {exc }}=360 \mathrm{~nm}^{[37]}$; c) Obtained from the onset of the fluorescence spectrum at $77 \mathrm{~K}$, which was determined by subtraction of the phosphorescence spectrum at $77 \mathrm{~K}$ from the steady-state $\mathrm{PL}$ spectrum at $77 \mathrm{~K}$; ${ }^{\mathrm{d}}$ Calculated from the onset of the phosphorescence spectra (delay: $2 \mathrm{~ms}$, window: $0 \mathrm{~ms}$ integration); ${ }^{\mathrm{e}} \Delta E_{\mathrm{ST}}=E\left(\mathrm{~S}_{1}\right)$ $\left.-E\left(\mathrm{~T}_{1}\right) ;{ }^{\mathrm{f}}\right)$ The HOMO and LUMO energies were determined using the relation $E_{\mathrm{HOMO} / \mathrm{LUMO}}=-\left(E^{\mathrm{ox}}{ }_{\text {onset }} / E^{\text {red }}{ }_{\text {onset }}+4.8\right) \mathrm{eV}$, ${ }^{[38]}$ where $E^{\text {ox }}$ and $E^{\text {red }}$ are the maxima of anodic and cathodic peak potentials, respectively calculated from DPV related to $\mathrm{Fc} / \mathrm{Fc}^{+} ;{ }^{\mathrm{g}}{ }_{\mathrm{H}-\mathrm{L}}=\left|E_{\mathrm{HOMO}}-E_{\mathrm{LUMO}}\right|$.

that leads to greater CT character and hence a lower oscillator strength for the transition. A modest blueshift in absorption is observed upon increasing solvent polarity for each emitter (Tables S12-S15 and Figure S63, Supporting Information). In order to probe this, solvent state specific calculations using the integral equation formalism variant of the polarizable continuum model were performed considering two solvents of significantly different polarity: $\mathrm{MeCN}$ and cyclohexane in order to determine the solvated excited energies. In these calculations, the electronic density of the emitters and the solvent reaction field are relaxed together self-consistently. This requires calculations of the individual $\mathrm{S}_{1-4}$ excited states. The observed hypsochromic shifts of these excited states is corroborated by these calculations, with both $\mathrm{S}_{3}$ and $\mathrm{S}_{4}$ showing an increase in energy moving from cyclohexane to $\mathrm{MeCN}$ (the $\mathrm{S}_{4}$ state of BIm(Me)PyPXZ being the exception where there is a modest redshift predicted from 4.49 to $4.47 \mathrm{eV}$ ). Much like the experimental data, the predicted changes are modest (cf. Table S8, Supporting Information).

The photophysical properties in toluene are summarized in Table 2 and the photoluminescence spectra shown in Figure 5b. The emission for each compound is broad and unstructured, indicative of an excited state with a dominant CT character. In line with computations, and consistent with the ground state optoelectronic characterization, the bluest emission at a photoluminescence maximum, $\lambda_{\mathrm{PL}}=497 \mathrm{~nm}(2.49 \mathrm{eV})$ occurs for $\operatorname{BIm}(t \mathrm{Bu})$ PyPXZ. BImPyPXZ shows the greenest emission at $513 \mathrm{~nm}(2.42 \mathrm{eV})$, followed by BIm(Ph)PyPXZ at $509 \mathrm{~nm}$ $(2.44 \mathrm{eV})$ and BIm(Me)PyPXZ at $501 \mathrm{~nm}(2.49 \mathrm{eV})$. The trends in emission maxima match those calculated in the DFT study, when considering the Boltzmann-averaged population of conformations for each emitter (Table 1). Positive solvatochromism was observed, corroborating the CT assignment for the emissive excited state (Figure S64 and Tables S12-S15, Supporting Information). The photoluminescence quantum yields in toluene, $\Phi_{\mathrm{PL}}$, range between $25 \%$ and $40 \%$. We speculate that the lowest $\Phi_{\mathrm{PL}}$ observed for $\mathrm{BIm}(t \mathrm{Bu}) \mathrm{PyPXZ}$ is associated with a higher nonradiative decay rate toward the ground state, in line with the broader distribution of conformations accessible at room temperature (see Figure 4). The $\Delta E_{\mathrm{ST}}$ values were determined from the difference in onset energies of the fluorescence and phosphorescence spectra recorded at $77 \mathrm{~K}$ (Figure S65, Supporting Information). $\Delta E_{\mathrm{ST}}$ values are larger for $\mathrm{BIm}(\mathrm{Me})$ PyPXZ, BIm(Ph)PyPXZ and BIm(tBu)PyPXZ, with values of $0.29,0.23$, and $0.33 \mathrm{eV}$, respectively, while for BImPyPXZ $\Delta E_{\mathrm{ST}}$ is much smaller at $0.12 \mathrm{eV}$ (Figure S65, Supporting Information, and Table 2). Although the values recorded are larger than those predicted using TDA-DFT, the trends are manifestly the same (Table 2). A minimal delayed component was observed in toluene. We attribute the low intensity of the Delayed Fluorescence, DF, in solution to collisional quenching (Figure S66a, Supporting Information).

With a view to fabricating OLEDs, mCP was selected as a suitable host material owing to its high triplet energy $(2.9 \mathrm{eV})$ and wide HOMO-LUMO gap (HOMO $=-6.1 \mathrm{eV}$, LUMO $=-2.4 \mathrm{eV}) .{ }^{[39]}$ For each emitter, a concentration screen was undertaken in order to determine the doping at which maximum $\Phi_{\mathrm{PL}}$ occurs (Figure S67, Supporting Information). BImPyPXZ displayed severe ACQ (possibly arising from its ability to form strong hydrogen bonds, which were observed in the crystal, Figure S42, Supporting Information) with an exponential decrease in photoluminescence quantum yield with increased doping, and so $1 \mathrm{wt} \%$ was used in order to maintain a high $\Phi_{\text {PL }}$ (Figure 6 and Table S17, Supporting Information). $N$-substitution mitigated somewhat ACQ and the highest values of $\Phi_{\mathrm{PL}}$ were obtained at $7 \mathrm{wt} \%$ doping concentration for the other three emitters (Figure 6 and Tables S18-S20, Supporting Information). The addition of bulky substituents to suppress ACQ is a known strategy. ${ }^{[40]}$ Photoluminescence quantum yields of $58 \%$, 68\%, and $49 \%$ were reported for $7 \mathrm{wt} \%$ films of BIm(Me)PyPXZ, BIm(Ph)PyPXZ, and BIm(tBu)PyPXZ, respectively, while the $\Phi_{\mathrm{PL}}$ is $60 \%$ for the $1 \mathrm{wt} \%$ doped film of BImPyPXZ. The emission maximum shifted bathochromically from 1 to $100 \mathrm{wt} \%$ doping [1779 $\mathrm{cm}^{-1}(0.221 \mathrm{eV})$ for BImPyPXZ, $1150 \mathrm{~cm}^{-1}(0.143 \mathrm{eV})$ for BIm(Me)PyPXZ, $1009 \mathrm{~cm}^{-1}(0.125 \mathrm{eV})$ for $\mathbf{B I m}(\mathbf{P h}) \mathbf{P y P X Z}$ and $923 \mathrm{~cm}^{-1}(0.114 \mathrm{eV})$ for $\mathbf{B I m}\left({ }^{t} \mathrm{Bu}\right)$ PyPX, Figure S68 and Table S17-S20, Supporting Information]. The trend in emission maxima of the 7 wt\% doped films in $\mathrm{mCP}$ is different to that observed in solution with $\mathbf{B I m}(\mathbf{P h})$ PyPXZ exhibiting the most redshifted emission $\left(\lambda_{\mathrm{PL}}=517 \mathrm{~nm}\right)$, followed by BImPyPXZ ( $\left.\lambda_{\mathrm{PL}}=513 \mathrm{~nm}\right)$, BIm(Me)PyPXZ $\left(\lambda_{\mathrm{PL}}=508 \mathrm{~nm}\right)$, and $\operatorname{BIm}(t \mathrm{Bu}) \operatorname{PyPXZ}\left(\lambda_{\mathrm{PL}}=501 \mathrm{~nm}\right)$, following the trends in conjugation length observed in the crystal structures with BIm(Ph)PyPXZ and BImPyPXZ being the most conjugated due to the smallest $\alpha$, followed by BIm(Me)PyPXZ and $\operatorname{BIm}\left({ }^{(} \mathrm{Bu}\right)$ PyPXZ (Table 3 and Figure 6). The emission lifetime of each emitter was recorded using time-resolved PL. The PL decays are displayed in Figure 7. In addition to the prompt decay, a delayed decay is visible from about 100 ns, i.e., from $0.1 \mu$ s onward. The delayed decay is not monoexponential as 

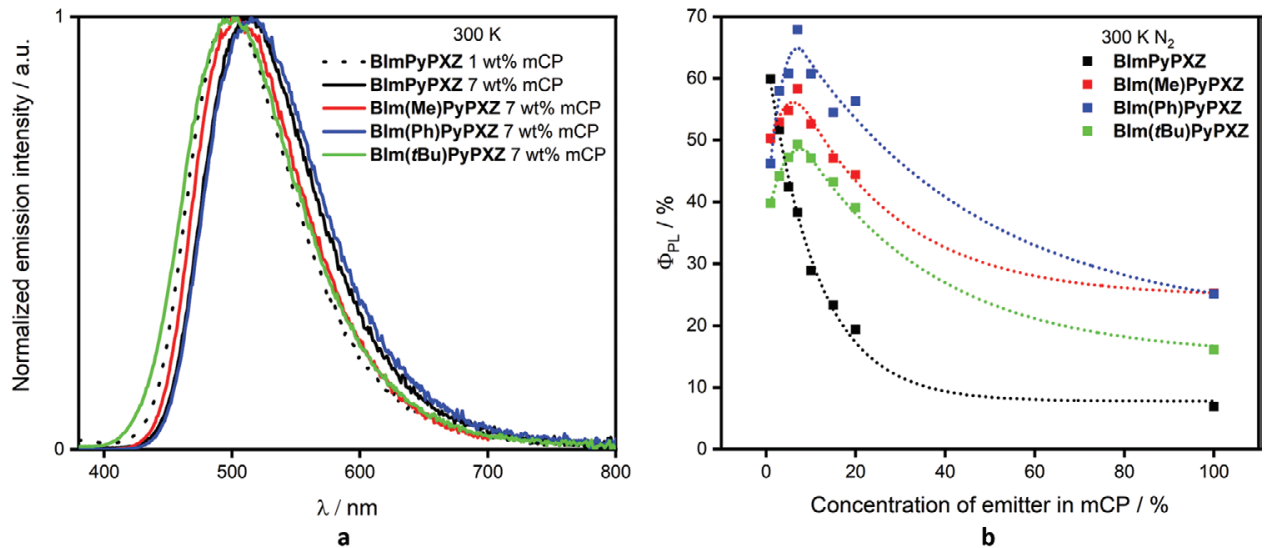

Figure 6. Solid-state photophysical data of the emitters. a) PL spectra of spin-coated doped $\mathrm{mCP}$ films. $\lambda_{\mathrm{exc}}=340 \mathrm{~nm}$. b) $\Phi_{\mathrm{PL}}$ at varying doping concentration of spin-coated mCP films under $\mathrm{N}_{2} . \lambda_{\text {exc }}=340 \mathrm{~nm}$, where fittings were applied to guide the reader to trends in $\Phi_{\mathrm{PL}}$.

we would expect from TADF behavior. Rather, its stretched-out nature indicates a superposition of a large number of decay times. We consider that this reflects the statistical distribution of torsion angles that prevails in a disordered thin film. There, molecules can be trapped in a kinetically frozen nonequilibrium geometry as opposed to a solution, where molecules can adopt their preferred equilibrium geometry. Correspondingly, the decay of the DF is not characterized by a single individual decay time, but rather by a mean value, calculated according to $\tau_{\mathrm{d} \text {,avg }}=\int I(t) \cdot t \mathrm{~d} t / \int I(t) \mathrm{d} t$ and listed in Table $3 .{ }^{[41]}$ The introduction of the substituent leads to an average lifetime that is about two to three times that of the unsubstituted parent compound BImPyPXZ. A comparison of the shortest monoexponential decay contribution in this distribution of lifetimes for each compound shows that the main effect of the substitution is the addition of more longer-lived components. This is consistent with the larger range of available torsion angles observed in the theoretical calculations and illustrated in Figure 4. The conformers with the larger torsion angles will also be characterized by lower wavefunction overlap between the donor and acceptor part and lower oscillator strength, hence longer lifetime.

The shortest exponential decays in the distribution are indicated by a blue line in Figure 7 and their overall contribution to the DF intensity is listed in Table 3. The value of the longest-lived component would depend on how low intensities one wishes to consider. The decays with an initial intensity fraction of $0.02 \%$ have lifetimes around $1 \mathrm{~ms}$ and are indicated by a green line in Figure 7 for reference. This distribution of lifetimes reflects a distribution of RISC rates. As detailed in the work by Gibson et al., ${ }^{[33]}$ the value of the RISC rate depends on the energy difference between ${ }^{3} \mathrm{CT}$ and ${ }^{3} \mathrm{LE}$ states (vibronic coupling between triplet states), and between ${ }^{1} \mathrm{CT}$ and ${ }^{3} \mathrm{LE}$ (spin-orbit coupling). In our compounds, a statistical distribution of torsion angles prevails, which implies a Gaussian distribution of the ${ }^{3} \mathrm{C}-\mathrm{T}^{3} \mathrm{LE}$ and ${ }^{1} \mathrm{C}-\mathrm{T}^{3} \mathrm{LE}$ energy differences. This results in a distribution of RISC rates, which manifests itself experimentally as a nonexponential decay of the delayed emission. If RISC rates are calculated on the basis of the measured lifetimes, then the average DF lifetimes imply average RISC rates in the range of $10^{4} \mathrm{~s}^{-1}$ for all compounds. The highest RISC rates obtained from the shortest lifetime contributions are around $5 \times 10^{5} \pm 1 \times 10^{5} \mathrm{~s}^{-1}$ for all compounds (see the Supporting Information for details). The shortest decays are about 10-60 times shorter than the average decay times and similar for all compounds except $\mathbf{B I m}(\boldsymbol{t B u}) \mathbf{P y P X Z}$, which is notably shorter at $6 \mu \mathrm{s}$. As this compound also has a lower $\Phi_{\mathrm{PL}}$, we attribute this particular shorter lifetime mainly to an increased nonradiative decay due to its higher torsional degree of freedom (cf. Figure 4). Superimposed spectra at $10 \mu \mathrm{s}$ and $1 \mathrm{~ms}$ were identical, which ruled out possible room temperature phosphorescence, which may have accounted for the long and variable lifetime at $300 \mathrm{~K}$ (Figure S71, Supporting Information).

The delayed emission is temperature dependent, showing the expected increase with rising temperature that is indicative of TADF (Figure 8). The $\Delta E_{\mathrm{ST}}$ values range between $0.21 \mathrm{eV}$ (for BIm(Me)PyPXZ) and $0.32 \mathrm{eV}$ [for $\operatorname{BIm}(t \mathrm{Bu})$ PyPXZ]. The

Table 3. Summary of the solid-state optoelectronic properties in spin-coated doped films in $\mathrm{mCP}$.

\begin{tabular}{|c|c|c|c|c|c|c|c|c|}
\hline Compound & $\lambda_{\mathrm{PL}}{ }^{\text {a) }}[\mathrm{nm}]$ & $\Phi_{\mathrm{PL}}{ }^{\text {b) }}[\%]$ & $\mathrm{S}_{1}^{\mathrm{c})}[\mathrm{eV}]$ & $\mathrm{T}_{1}^{\mathrm{c})}[\mathrm{eV}]$ & $\Delta E_{\mathrm{ST}}{ }^{\mathrm{d})}[\mathrm{eV}]$ & $\tau_{\mathrm{p}}^{\mathrm{e})}[\mathrm{ns}]$ & $\tau_{\mathrm{d} \text {,avg }}{ }^{\mathrm{f}}[\mu \mathrm{s}]$ & $\tau_{\text {short }}{ }^{\mathrm{g})}[\mu \mathrm{s}]$ \\
\hline BImPyPXZh) & $501\left(513^{i)}\right)$ & 59.3 & 2.77 & 2.56 & 0.21 & 10.1 & 260 & $27(44 \%)$ \\
\hline $\left.\operatorname{BIm}(M e) P y P X Z^{i}\right)$ & 508 & 58.3 & 2.79 & 2.56 & 0.23 & 10.0 & 770 & $34(25 \%)$ \\
\hline $\operatorname{BIm}(P h) P y P X Z^{i)}$ & 517 & 67.9 & 2.76 & 2.55 & 0.21 & 10.8 & 440 & $17(30 \%)$ \\
\hline $\left.\operatorname{BIm}(t B u) P y P X Z^{i}\right)$ & 501 & 49.3 & 2.95 & 2.62 & 0.33 & 7.9 & 460 & $7.0(21 \%)$ \\
\hline
\end{tabular}

a) $\lambda_{\text {exc }}=340 \mathrm{~nm}$; b) Determined using an integrating sphere. $\lambda_{\text {exc }}=340 \mathrm{~nm}$; ${ }^{c} \mathrm{~S}_{1}$ and $\mathrm{T}_{1}$ calculated from onset of the fluorescence spectrum at $77 \mathrm{~K}$ and the phosphorescence spectrum (delay: $2 \mathrm{~ms}$; window: $10 \mathrm{~ms}$ integration) at $77 \mathrm{~K}$, where the fluorescence was inferred by subtracting the phosphorescence spectrum from the steady-state PL

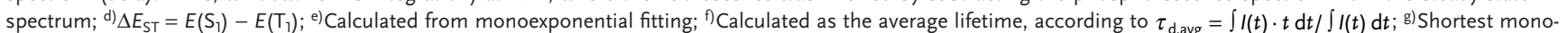
exponential component of the lifetime distribution, along with its contribution to the overall DF signal in \%; ${ }^{h)} 1 \mathrm{wt} \%$ emitter in $\mathrm{mCP}$; i) 7 wt\% emitter in $\mathrm{mCP}$. 

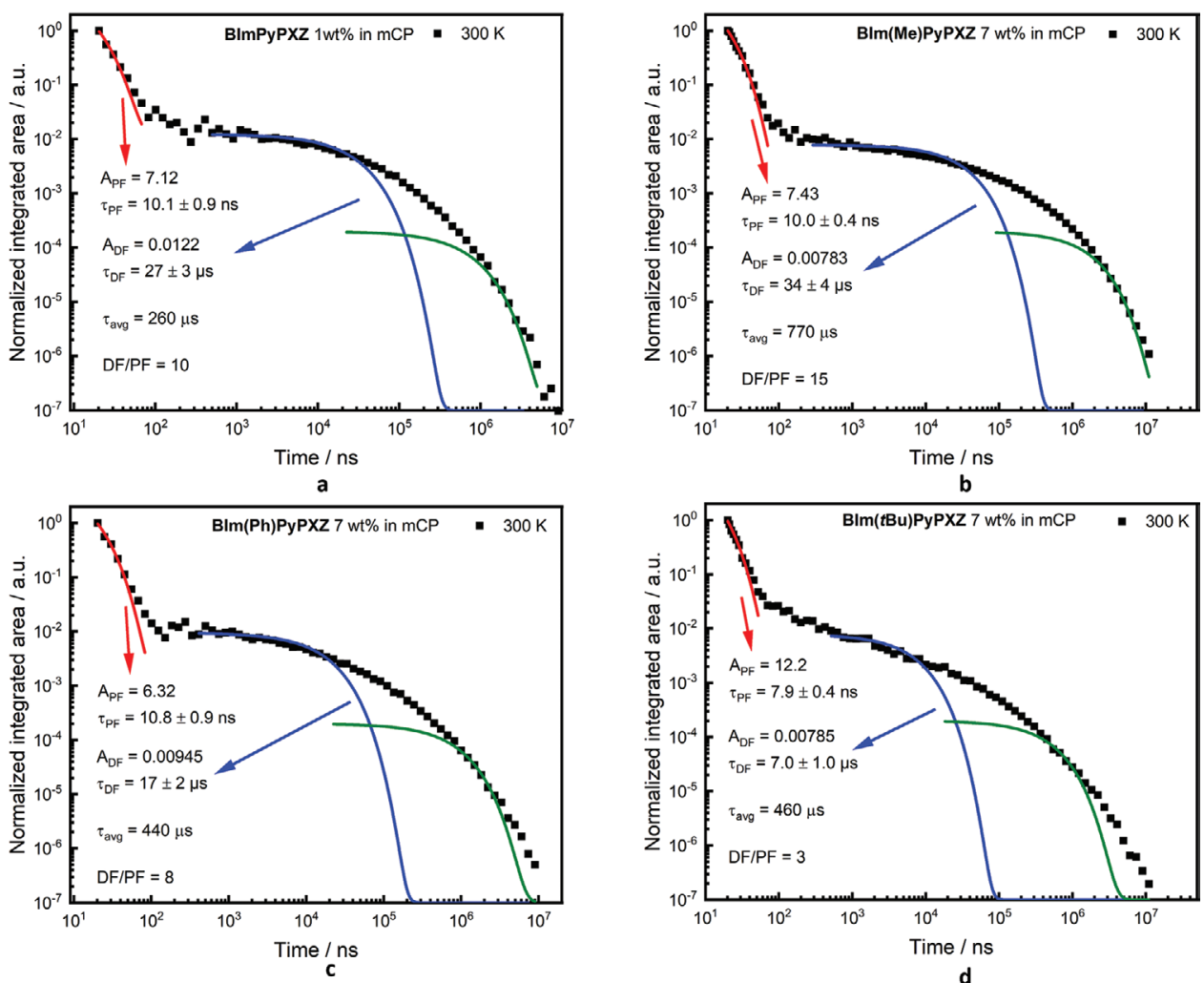

Figure 7. Room temperature PL decay of a) BImPyPXZ; b)BIm(Me)PXZ; c) BIm(Ph)PXZ; d) BIm(tBu)PXZ in mCP. Also shown are the prompt decay (red line), shortest monoexponential fit to the $D F$ that is possible, $I(t)=A_{D F} \exp \left(-t / \tau_{D F}\right)$, as a blue line along with the pertinent fit parameters. The longest possible monoexponential fits with $A_{D F}=0.0002$ is also given as green line for reference.

differences in trends for $\Delta E_{\mathrm{ST}}$ in $\mathrm{mCP}$ compared to both toluene and DFT calculations are likely due to different populations of conformers in each medium.

\subsection{Device Performance}

Owing to their promising photophysical behavior, vacuumdeposited OLEDs were fabricated for BImPyPXZ, BIm(Me) PyPXZ, and BIm(Ph)PyPXZ. Due to the instability of the emitter upon sublimation, devices containing $\operatorname{BIm}(t \mathbf{B u})$ PyPXZ were not prepared. The optimized device stack used was: indium tin oxide (ITO)/4,4'-cyclohexylidenebis[ $N$, $N$-bis(4methylphenyl)benzenamine] (TAPC) $(40 \mathrm{~nm}) /$ tris (4-carbazoyl9-ylphenyl)amine (TCTA) (10 nm)/emitter: mCP $(30 \mathrm{~nm}) /$ bis[2-(diphenylphosphino)phenyl]ether oxide (DPEPO) $(5 \mathrm{~nm}) /$ $\mathrm{TmPyPb}(50 \mathrm{~nm}) / \mathrm{LiF}(1 \mathrm{~nm}) / \mathrm{Al}$, where ITO is the anode, TAPC and TCTA act as hole-transporting layers, 1,3-bis( $N$-carbazolyl) benzene $(\mathrm{mCP})$ is the host, DPEPO acts as a hole-blocking layer, 1,3,5-tri( $m$-pyridin-3-ylphenyl)benzene (TmPyPB) is the electron-transporting material, and LiF modifies the work function of the aluminum cathode (Figure 9). Four devices are presented where device 1a contains $1 \mathrm{wt} \%$ BImPyPXZ, device 2a contains 7 wt\% BIm(Me)PyPXZ, device 3a contains $7 \mathrm{wt} \%$ BIm(Ph)PyPXZ and device 4a contains 1 wt\% BIm(Me) PyPXZ, the latter of which was fabricated to assess the impact of doping concentration on the performance of the OLED. Another series of devices that used $40 \mathrm{~nm} \mathrm{TmPyPb}$ were prepared with lower efficiencies and are presented in the Supporting Information, designated as device series b (Table S22 and Figures S72 and S73, Supporting Information). The performance metrics of the OLEDs are summarized in Table 4.

The electroluminescence spectra are shown in Figure 10a and the corresponding Commision International de l'Éclairage (CIE) coordinates plotted in Figure 10b. The electroluminescence maxima, $\lambda_{\mathrm{EL}}$, match well with the corresponding $\lambda_{\mathrm{PL}}$. Device 3a, containing $\operatorname{BIm}(\mathbf{P h}) \mathbf{P y P X Z}$, shows the most redshifted emission $\left(\lambda_{\mathrm{EL}} 518 \mathrm{~nm}\right)$. Excellent $\mathrm{EQE}_{\max }$ were reported for the series of $18.6 \%, 23.9 \%$, and $22.2 \%$, respectively, for BImPyPXZ (1 wt \% mCP), BIm(Me)PyPXZ (7 wt\% mCP), and BIm(Ph)PyPXZ (7 wt \% mCP) (Figure 10c). As the previous $\Phi_{\mathrm{PL}_{\mathrm{L}} \mathrm{S}}$ were recorded with spin-coated films, the $\Phi_{\mathrm{PL}}$ of vacuum-evaporated films were also measured (1 wt\% BImPyPXZ and $7 \mathrm{wt} \%$ $\operatorname{BIm}(\mathrm{Me}) \mathbf{P y P X Z}$ and $\mathbf{B I m}(\mathbf{P h}) \mathbf{P y P X Z}$ in $\mathrm{mCP})$, with values of $63 \%, 69 \%$, and $62 \%$, respectively. These values are similar to those obtained for spin-coated films $(60 \%, 58 \%$, and $68 \%$, respectively); small differences are likely due to differences in packing in the films as a result of the two processes. ${ }^{[20]}$ Considering these values and assuming 100\% charge recombination and a maximum outcoupling efficiency of $30 \%$, the theoretical maximum EQEs ( $\mathrm{EQE}_{\max }$ ) for devices 1a, 2a, and 3a are $\approx 19 \%$, $\approx 21 \%$, and $\approx 19 \%$ respectively, indicating $100 \%$ exciton utilization efficiency and confirming the operation of TADF in the device. Each of the devices shows moderate efficiency roll-off at $100 \mathrm{~cd}$ $\mathrm{m}^{-2}$ of $24 \%, 19 \%$, and $13 \%$ of the $\mathrm{EQE}_{\max }$ for devices $1 \mathrm{a}, 2 \mathrm{a}$, and 3a, respectively. There is a more severe efficiency roll-off at 

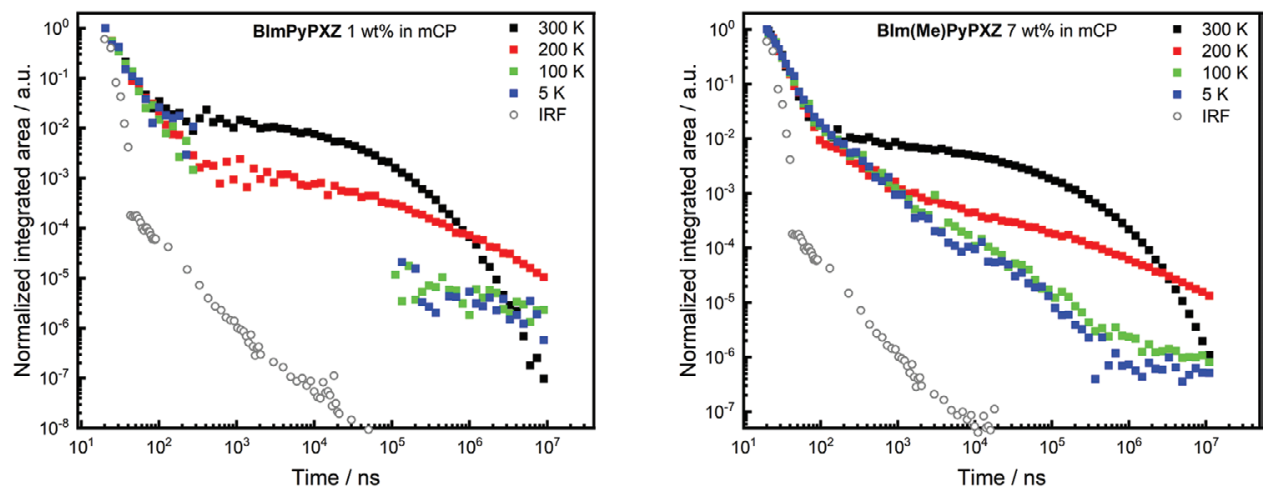

a

b
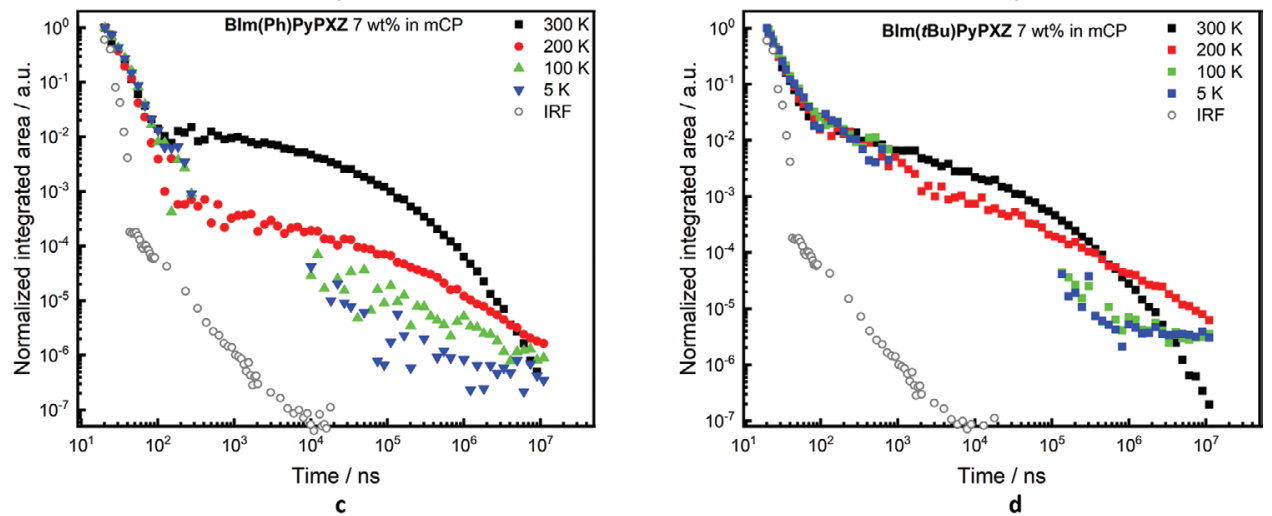

Figure 8. Temperature-dependent time-resolved PL in doped $\mathrm{mCP}$ films, $\lambda_{\text {exc }}=355 \mathrm{~nm}$. a) $1 \mathrm{wt} \% \mathrm{BImPyPXZ}$ in $\mathrm{mCP}$. b) $7 \mathrm{wt} \%$ BIm(Me)PyPXZ in mCP. c) $7 \mathrm{wt} \% \mathrm{BIm}(\mathrm{Ph})$ PyPXZ in $\mathrm{mCP}$. d) $7 \mathrm{wt} \% \mathrm{BIm}(\mathrm{tBu}) \mathbf{P y P X Z}$ in $\mathrm{mCP}$. IRF is the instrument response function.

$1000 \mathrm{~cd} \mathrm{~m}^{-2}$, with $\mathrm{EQE}_{1000}$ decreasing to $6.3 \%, 11.7 \%$, and $9.6 \%$, respectively. This is due to the increased number of charge carriers, increasing the likelihood of annihilation pathways, with the long lifetimes of the triplet state exacerbating this issue. We next compared the effect of doping concentration on the device performance (devices 2a and 4a) where the emitter, BIm(Me) PyPXZ, was doped into the $\mathrm{mCP}$ film at 7 and $1 \mathrm{wt} \%$, respectively. There is a slight redshift apparent upon increased doping that is similar to the effect observed for increased doping on the $\lambda_{\mathrm{PL}}$ (Table 3), shifting from 501 to $511 \mathrm{~nm}$ when doping is

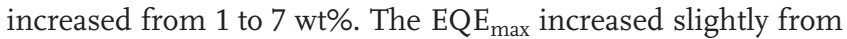
$21.4 \%$ to $23.9 \%$ upon increasing the doping, in line with the $\Phi_{\text {PL }}$ data (Table S7, Supporting Information). The improved exciton harvesting at higher brightness becomes apparent when considering the maximum luminance, which is doubled for devices with $7 \mathrm{wt} \%$ doping, nearing $18000 \mathrm{~cd} \mathrm{~m} \mathrm{~m}^{-2}$, compared to the devices with the emitter at $1 \mathrm{wt} \%$ doping where it was $\approx 8000 \mathrm{~cd} \mathrm{~m}^{-2}$. A similar value was achieved for the $1 \mathrm{wt} \%$

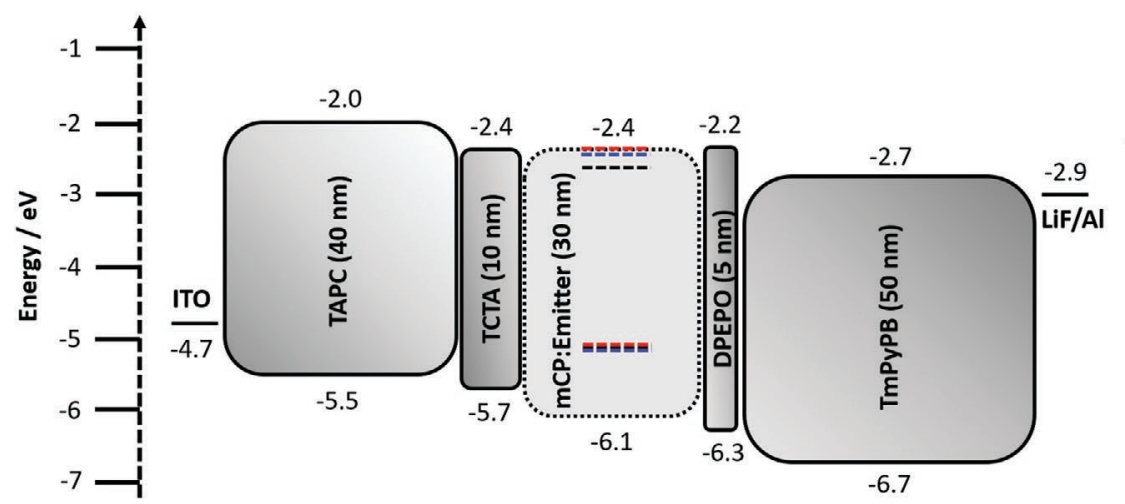

ImidPyPXZ; HOMO -5.16 eV, LUMO -2.60 eV
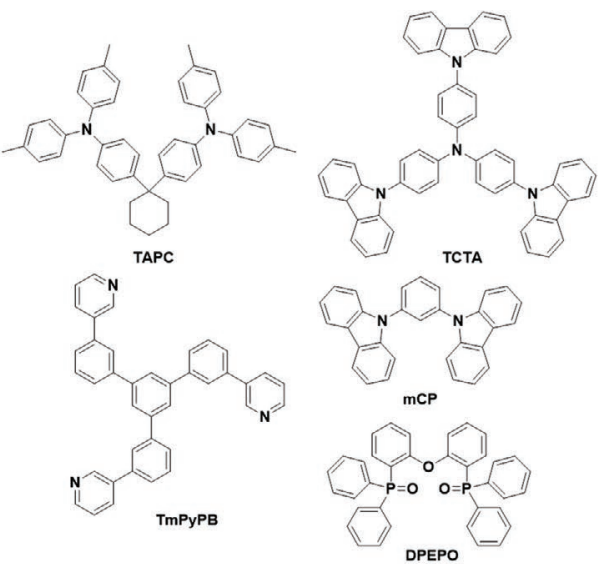

Imid(Me)PyPXZ; HOMO -5.16 eV, LUMO -2.37 eV

Imid(Ph)PyPXZ; HOMO -5.18 eV, LUMO -2.40 eV

Figure 9. Device architecture including energy levels for device series a and structures of materials used. 
Table 4. Device properties of device series a of the various emitters in vacuum evaporated OLEDs.

\begin{tabular}{|c|c|c|c|c|c|c|c|}
\hline $\begin{array}{l}\text { Compound } \\
\text { (device/emitter concentration) }\end{array}$ & $\begin{array}{l}V_{\text {on }} \\
{[V]}\end{array}$ & $\begin{array}{c}\lambda_{\mathrm{EL}} \\
{[\mathrm{nm}]}\end{array}$ & $\begin{array}{c}\mathrm{EQE}_{\max } ; \mathrm{EQE}_{100} ; \mathrm{EQE}_{1000} \\
{[\%]}\end{array}$ & $\begin{array}{l}\mathrm{Cd}_{\max } \\
{[\mathrm{A}]}\end{array}$ & $\begin{array}{l}\operatorname{Im}_{\max } \\
{[\mathrm{W}]}\end{array}$ & $\begin{array}{l}\operatorname{Lum}_{\max } \\
{\left[\mathrm{cd} \mathrm{m}^{-2}\right]}\end{array}$ & CIE $(8 \mathrm{~V})$ \\
\hline BImPyPXZ (1a/1 wt\%) & 3.5 & 508 & $18.6 ; 14.1 ; 6.3$ & 50 & 42 & 8355 & $0.23,0.47$ \\
\hline BIm(Me)PyPXZ (2a/7 wt\%) & 3.3 & 511 & $23.9 ; 19.3 ; 9.6$ & 68 & 62 & 17717 & $0.25,0.51$ \\
\hline BIm(Ph)PyPXZ (3a/7 wt\%) & 3.3 & 518 & $22.2 ; 19.3 ; 11.7$ & 67 & 60 & 21227 & $0.28,0.54$ \\
\hline $\operatorname{BIm}(\mathrm{Me}) \operatorname{PyPXZ}(4 \mathrm{a} / 1 \mathrm{wt} \%)$ & 3.6 & 501 & $21.4 ; 15.7 ; 6.6$ & 54 & 45 & 7697 & $0.21,0.44$ \\
\hline
\end{tabular}
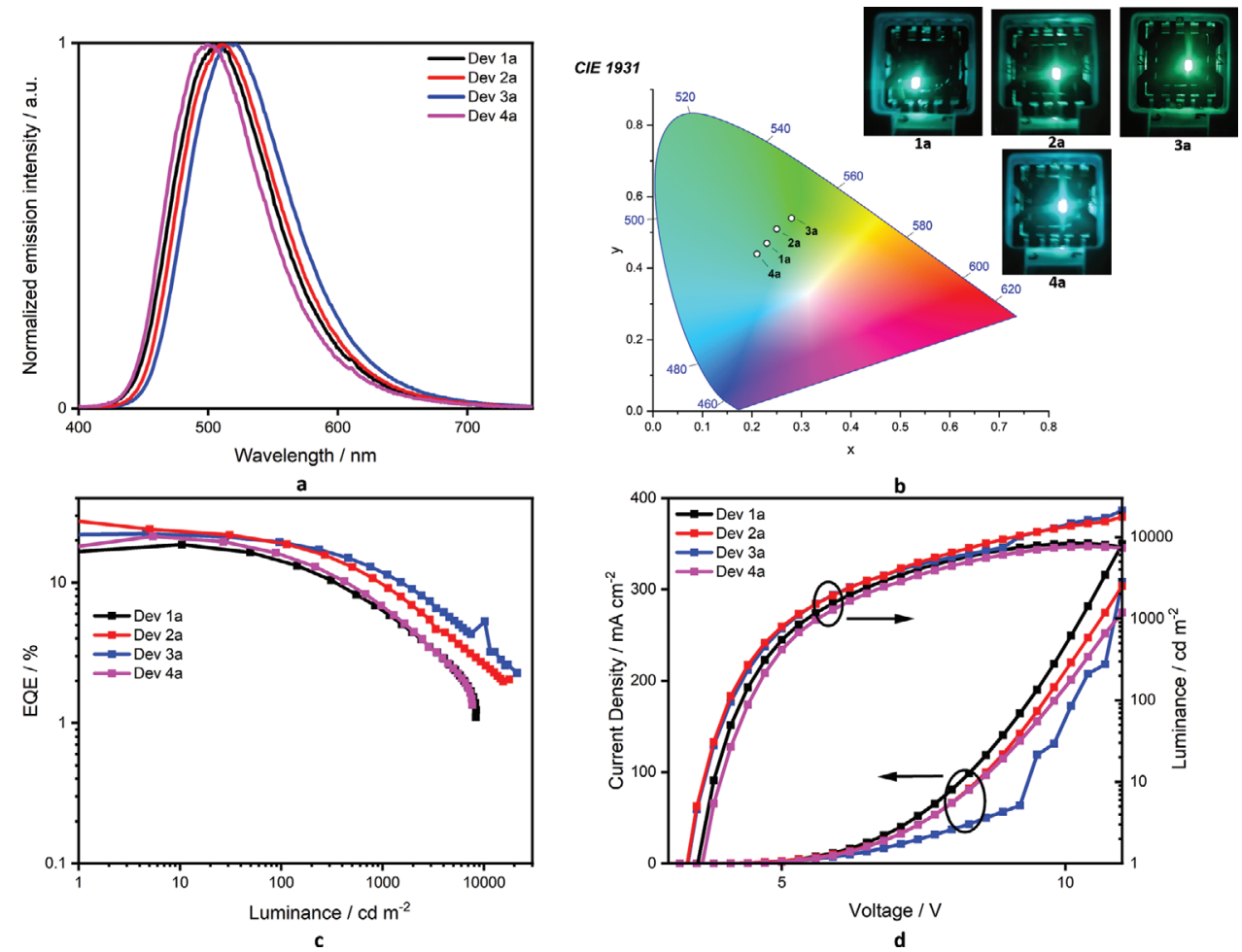

Figure 10. OLED device series a. a) Electroluminescence spectra. b) CIE coordinates. c) External quantum efficiency versus luminance. d) Current density/luminance versus voltage.

BImPyPXZ (device 1a) at around $8000 \mathrm{~cd} \mathrm{~m}^{-2}$. The device using BIm(Ph)PyPXZ showed the greatest $l_{\text {umax }}$, at more than $21000 \mathrm{~cd} \mathrm{~m}^{-2}$.

\section{Conclusions}

We have introduced a new acceptor unit, pyridylbenzimidazole into the donor-acceptor TADF lexicon, in which varying the $N$-substitution of the benzimidazole modulates both the emission energy and mitigates against ACQ. The bulkier the $\mathrm{N}$-substituent, the larger the torsion between the pyridine and benzimidazole rings. This has a direct consequence on $\Delta E_{\mathrm{ST}}$ and the emission color, with minimal impact on RISC efficiency, which is also facilitated by the presence of intermediate triplet states. Efficient OLEDs were fabricated, with EQE ${ }_{\max }$ surpassing 20\% for BIm(Me)PyPXZ and BIm(Ph)PyPXZ. Functionalized derivatives allowed for high-performance OLEDs to be fabricated at increased doping, resulting in devices showing much higher maximum luminance values. These results illustrate a family of high-performance TADF emitters.

\section{Supporting Information}

Supporting Information is available from the Wiley Online Library or from the author.

\section{Acknowledgements}

The St Andrews team would like to thank the Leverhulme Trust (RPG2016-047) for financial support. P.R. acknowledges support from a Marie Skłodowska-Curie Individual Fellowship (MCIF; No. 749557). S.M.S. acknowledges support from the Marie Skłodowska-Curie Individual Fellowship, Grant Agreement No. 838885 (NarrowbandSSL). Computational resources have been provided by the Consortium des Équipements de Calcul Intensif (CÉCI), funded by the Fonds de la Recherche Scientifiques de Belgique (F.R.S.-FNRS) under Grant No. 2.5020 .11 as well as the Tier-1 supercomputer of the Fédération Wallonie-Bruxelles, 
infrastructure funded by the Walloon Region under the Grant Agreement No. 1117545. The authors acknowledge support from the European Union's Horizon 2020 research and innovation programme under the Innovative Training Network TADFlife (No. 812872). Y.O. acknowledges funding by the Fonds de la Recherche Scientifique-FNRS under Grant No. F.4534.21 (MIS-IMAGINE). D.B. is an FNRS Research Director.

\section{Conflict of Interest}

The authors declare no conflict of interest.

\section{Data Availability Statement}

The data that support the findings of this study are openly available in the University of St Andrews repository at https://doi. org/10.17630/19a1218e-1aea-4331-b65b-103b15b9699c.

\section{Keywords}

DFT calculations, organic light-emitting diodes, photophysics, pyridylbenzimidazole, thermally activated delayed fluorescence

Received: April 27, 2021

Revised: June 19, 2021

Published online:

[1] M. Y. Wong, E. Zysman-Colman, Adv. Mater. 2017, 29, 1605444.

[2] Y. Liu, C. Li, Z. Ren, S. Yan, M. R. Bryce, Nat. Rev. Mater. 2018, 3, 18020.

[3] Z. Yang, Z. Mao, Z. Xie, Y. Zhang, S. Liu, J. Zhao, J. Xu, Z. Chi, M. P. Aldred, Chem. Soc. Rev. 2017, 46, 915.

[4] H. Uoyama, K. Goushi, K. Shizu, H. Nomura, C. Adachi, Nature 2012, 492, 234.

[5] R. G. Brown, N. Entwistle, J. D. Hepworth, K. W. Hodgson, B. May, J. Phys. Chem. 1982, 86, 2418.

[6] F. R. Prieto, M. Mosquera, M. Novo, J. Phys. Chem. 1990, 94, 8536.

[7] G. S. Yellol, J. G. Yellol, V. B. Kenche, X. M. Liu, K. J. Barnham, A. Donaire, C. Janiak, J. Ruiz, Inorg. Chem. 2015, 54, 470.

[8] Y. Qu, C. Wang, K. Zhao, Y. Wu, G. Huang, X. Han, H. Wu, J. Coord. Chem. 2019, 72, 3046.

[9] T. Jella, M. Srikanth, R. Bolligarla, Y. Soujanya, S. P. Singh, L. Giribabu, Dalton Trans. 2015, 44, 14697.

[10] A. M. Mansour, K. Radacki, Dalton Trans. 2020, 49, 4491.

[11] V. V. Pavlishchuk, A. W. Addison, Inorg. Chim. Acta 2000, 298, 97.

[12] K. C. Dash, H. Mohanta, J. Inoorg. Nucl. Chem. 1978, 40, 499.

[13] W.-C. Chen, Z.-L. Zhu, C.-S. Lee, Adv. Opt. Mater. 2018, 6, 1800258.

[14] Z. Huang, S. Xiang, Q. Zhang, X. Lv, S. Ye, R. Guo, L. Wang, J. Mater. Chem. C 2018, 6, 2379.
[15] Q. D. Liu, M. S. Mudadu, R. Thummel, Y. Tao, S. Wang, Adv. Funct. Mater. 2005, 15, 143.

[16] T. Teng, K. Li, G. Cheng, Y. Wang, J. Wang, J. Li, C. Zhou, H. Liu, T. Zou, J. Xiong, C. Wu, H.-X. Zhang, C.-M. Che, C. Yang, Inorg. Chem. 2020, 59, 12122.

[17] J.-H. Jia, D. Liang, R. Yu, X.-L. Chen, L. Meng, J.-F. Chang, J.-Z. Liao, M. Yang, X.-N. Li, C.-Z. Lu, Chem. Mater. 2020, 32, 620.

[18] T. Shan, Z. Gao, X. Tang, X. He, Y. Gao, J. Li, X. Sun, Y. Liu, H. Liu, B. Yang, P. Lu, Y. Ma, Dyes Pigm. 2017, 142, 189

[19] Z. Huang, B. Wang, Q. Zhang, S. Xiang, X. Lv, L. Ma, B. Yang, Y. Gao, L. Wang, Dyes Pigm. 2017, 140, 328

[20] D. Hall, S. M. Suresh, P. L. dos Santos, E. Duda, S. Bagnich, A. Pershin, P. Rajamalli, D. B. Cordes, A. M. Z. Slawin, D. Beljonne, A. Köhler, I. D. W. Samuel, Y. Olivier, E. Zysman-Colman, Adv. Opt. Mater. 2020, 8, 1901627.

[21] J. Mei, N. L. C. Leung, R. T. K. Kwok, J. W. Y. Lam, B. Z. Tang, Chem. Rev. 2015, 115, 11718.

[22] Y.-S. Lee, Y.-H. Cho, S. Lee, J.-K. Bin, J. Yang, G. Chae, C.-H. Cheon, Tetrahedron 2015, 71, 532

[23] H. Tanaka, K. Shizu, H. Miyazaki, C. Adachi, Chem. Commun. 2012, 48, 11392.

[24] M. K. Etherington, F. Franchello, J. Gibson, T. Northey, J. Santos, J. S. Ward, H. F. Higginbotham, P. Data, A. Kurowska, P. L. Dos Santos, D. R. Graves, A. S. Batsanov, F. B. Dias, M. R. Bryce, T. J. Penfold, A. P. Monkman, Nat. Commun. 2017, 8, 14987.

[25] M. Moral, L. Muccioli, W. J. Son, Y. Olivier, J. C. Sancho-García, J. Chem. Theory Comput. 2015, 11, 168.

[26] H. Sun, C. Zhong, J.-L. Brédas, J. Chem. Theory Comput. 2015, 11, 3851.

[27] C. Li, D. Li, Y. Shi, Y. Liu, Org. Electron. 2018, 54, 177.

[28] M. Tian, C. Wang, L. Wang, K. Luo, A. Zhao, C. Guo, Luminescence 2014, 29, 540 .

[29] M. A. El-Sayed, Acc. Chem. Res. 1968, 1, 8 .

[30] M. K. Etherington, J. Gibson, H. F. Higginbotham, T. J. Penfold, A. P. Monkman, Nat. Commun. 2016, 7, 13680.

[31] T. Hosokai, H. Matsuzaki, H. Nakanotani, K. Tokumaru, T. Tsutsui, A. Furube, K. Nasu, H. Nomura, M. Yahiro, C. Adachi, Sci. Adv. 2017, 3, e1603282.

[32] C. M. Marian, J. Phys. Chem. C 2016, 120, 3715.

[33] J. Gibson, A. P. Monkman, T. J. Penfold, ChemPhysChem 2016, 17, 2956.

[34] Y. Olivier, B. Yurash, L. Muccioli, G. D'Avino, O. Mikhnenko, J. C. Sancho-García, C. Adachi, T. Q. Nguyen, D. Beljonne, Phys. Rev. Mater. 2017, 1, 075602.

[35] F. B. Dias, J. Santos, D. R. Graves, P. Data, R. S. Nobuyasu, M. A. Fox, A. S. Batsanov, T. Palmeira, M. N. Berberan-Santos, M. R. Bryce, A. P. Monkman, Adv. Sci. 2016, 3, 1600080.

[36] Y. Olivier, M. Moral, L. Muccioli, J.-C. Sancho-García, J. Mater. Chem. C 2017, 5, 5718

[37] W. H. Melhuish, J. Phys. Chem. 1961, 65, 229.

[38] C. M. Cardona, W. Li, A. E. Kaifer, D. Stockdale, G. C. Bazan, Adv. Mater. 2011, 23, 2367.

[39] S. H. Kim, J. Jang, J. Y. Lee, Appl. Phys. Lett. 2007, 90, 223505.

[40] K. Trofymchuk, A. Reisch, I. Shulov, Y. Mély, A. S. Klymchenko, Nanoscale 2014, 6, 12934.

[41] J. R. Lakowicz, Principles of Fluorescence Spectroscopy, Springer, New York 2007. 